7

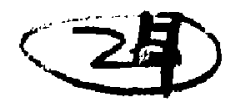

$D R \cdot 1829-5$

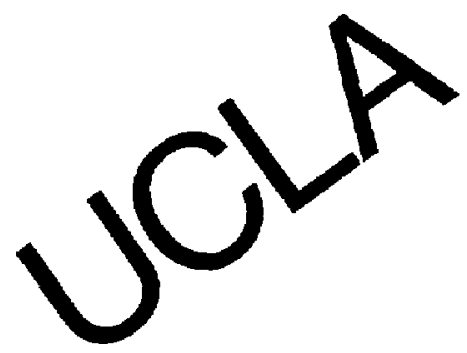

UCLA-ENG-8611

PPG-937

Materials Data Base and Design Equations

For the UCLA Solid Breeder Blanket

Shahram Sharafat, Robert Amodeo and Nasr M. Ghoniem

February 1986

1

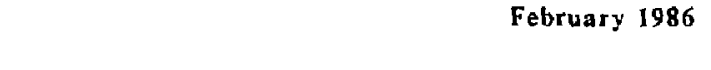

1

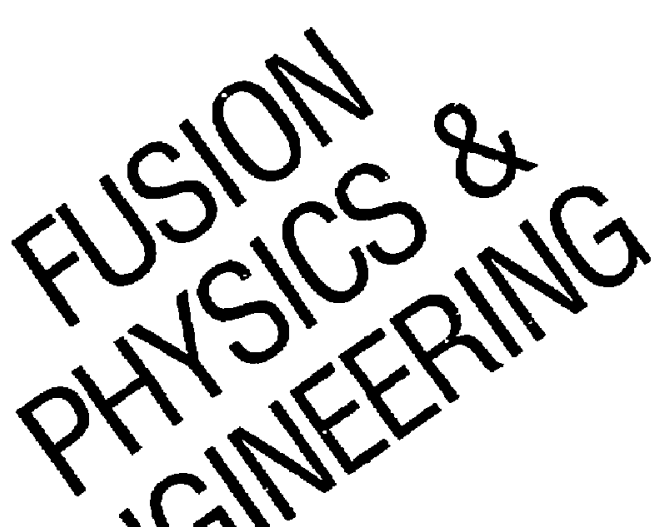

DISTRIBUTION OF THIS DOCUMENT !S UNUMITED

MECHANICAL. AEROSPACe: AND NUCLEAR ENGINEERING DEPT.

AND

CENTER FOR PLASMA PHYSICS AND FUSION ENGINEERING

UNIVERSITY OF CALIFORNIA. LOS ANGELES 
DCLA-ENG -8611

DE86 012971

UCLA-ENG-8611

PPG-937

\title{
Materials Data Base and Design Equations For the UCLA Solid Breeder Blanket
}

\author{
Shahram Sharafat, Robert Amodeo and Nasr M. Gboniem
}

February 1986

This work was supported by the U.S. Department of Energy, Office of Fusion Energy, Grant \#DE- 03-80ER52061, with UCLA.

\section{DISCLAIMER}

This repon was prepared as an accounl of work sponsored by an agency of the United States Goversment. Neither the United States Cryernment nor any agency thereuf, nor any of their employecs, makes any warranty, express or implied, or assumes any legal fiabitity or responsibility for the aceuracy, completeness, or usefulness of any information, apparatus, product, or process disclased, or represents that its use would not inftinge privately owned rights. Refesence herein to any specilic commercial product, process, or service by trade mame, trademark, manufacturer, of otherwise does not necessarily constitute or imply its endorsement, recommendation, or favering by the United States Government or any agency thereof. The views and opinions of Julhors expressed herein do not necessarity state or reflect those of the United States Government or any agency thereof. 


\section{DISGLATHER}

This report was prepared as an account of work sponsored by an ageccy of the Untted States Covernment. Nefther the United States Government nor any agency thereof, nor any of their employees, nakes any warranty, express or tmplled, or asgunes any legal 11 abt11ty or responstb111ty for the accuracy, completeness, or usefulness of any Information, apparatus, product, or process disclosed, or represents that its use would not infinge privately owned Itghts. Reference hereln to any specific conmerctal product, process, or service by trade name, trademark, manufacturer, or otherwise, does not necessarily constitute or 1mply fts endorsement, recommendation, or favoring by the Untted States Government or any agency thereof. The views and opinions of authors expressed hexeln do not necessar1ly otate or reflect thoge of the United States Government or any agency thereof. 
TABLE OF CONTENTS

Page \#

1. Introduation ........................ . 1

2. Material Properties . . . . . . . . . . . . . . . 2

3. Phenomenological and Empirical Equations . . . . . . . 4

3.1 Ferritic Steels: HT-9, 2 1/4 CI - 1 Mo . . . . . 5

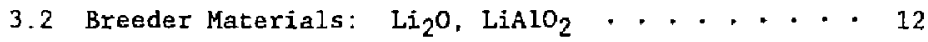

3.3 Selected Placs for Material Properties . . . . . . 22

4. Phenomenological Swelling Equations for Solid Breeder . and Neution Multiplier Materials . . . . . . . 33

4.1 Theory ................ . . 33

4.2 Swelling of Solid Breeder Material . . . . . . 37

4.3 Beryllium Swelling . . . . . . . . . . . . 43

5. Sintering Phenomena in Breeder Ceranics . . . . . . . . . 52

5.1 Introduction . . . . . . . . . . . . . . 52

5.2 The Effect of Porosity . . + . . . . . . . . 53

5.3 Sincering. . . . . . . . . . . . . . 56

Acknowledgements .. . . . . . . . . . 61

References........................ 62 
1. Helium retention in the FUBR-IA experiment (after Hollenberg [2]) . . 34

2. Dfametrical swelling of $\mathrm{LI}_{2} \mathrm{O}, \mathrm{LI}_{4} \mathrm{StO}_{4}, \mathrm{LI}_{2} \mathrm{ZrO}_{3}$ and $\mathrm{LiAlO}_{2}$ after achieving a burnup of $3 \times 10^{20}$ captures/ $\mathrm{cm}^{3}$ (after hollenberg [2]) . . 35

3. Volumetrfe swelling of $\mathrm{LiAlO}_{2}$ exposed to $\simeq 5 \mathrm{MW} / \mathrm{m}^{2}$ of neutron wall loading as a function of irradiation time . . . . . . . . 39

4. Volumetric swelling of $\mathrm{Li}_{2} O$ exposed to $5 \mathrm{MN} / \mathrm{m}^{2}$ of neutron wall wall loading as a function of irradiation time . . . . . . . . 42

5. Blanket pin average steady state temperature as a function of distance from the iirst wall for a neutron wall loading of $=5 \mathrm{MW} / \mathrm{m}^{2}$...................... 45

6. Helium production in $\mathrm{LiAlO}_{2}$ breeder pins as a function of distance from the first wall . . . . . . . . . . . . . . . . 47

7. Average helium production in Be-rods as a function of distance from the first wall .................. 48

8. Average volumetric swelling of $\mathrm{LiAlO}_{2}$ breeder cods exposed to $5 \mathrm{MW} / \mathrm{m}^{2}$ neutron wall loading after 3 years of irradiation..... 49

9. Dolumetric swelling of Be-rods exposed to $5 \mathrm{MW} / \mathrm{m}^{2}$ neutron wall loading after 1,2 , and 3 years of Irradiation ......... 50

\section{TABLES}

1. Sumary for blanket material properties current status (12/85) . . . 4

2. Estimates for bubble number densities and radii using above model

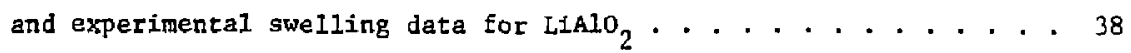

3. Experimental and analytical swelling results for $\mathrm{LiAlO}_{2}$ for $500 \leq \mathrm{T} \leq 900^{\circ} \mathrm{C} \ldots \ldots . \ldots . \ldots . \ldots$

4. Experimental sw5lling and helium retention data for $\mathrm{L}_{2} \mathrm{O}$. . . . . 40 
5. Estimate for bubbie number denstties and radii using the above model and experdmental data for $\mathrm{Li}_{2} \mathrm{O}$. . . . . . . . . . . 40

6. Experimental and analycical swelling results for $\mathrm{Li}_{2} \mathrm{O}$ for $500<\mathrm{T}<900^{\circ} \mathrm{C} . . . . . . . . . . . . . .441$

7. Experimental and analytical swelling results for Be irradiated to 50,000 appm at $400^{\circ}<\mathrm{T}<600^{\circ} \mathrm{C} \ldots \ldots 42$ 


\section{MATERIALS DATA BASE AND DESIGN EQUATIONS}

\section{Introduction}

The need for a complete and coherent materlal data base for fuston reactor systems has been an important issue for some time now. Since the choices for materials used in fusion reactors are becoming more apparent, it is important to be able to quickly access this data to facilitate reactor design. The problem with the current choices of materials is that there is not enough data available to supply complete information on all the important material properties under anticipated fusion operating conditions. In piaces where there is a deficit in data, it is therefore necessary to extrapolate informacion to support analysis which covers ranges of undetermined values.

The philosophy of a data base is one of expansion and modification. This will lead to a constantly growing collection of most recently acquired information. Based on this philosophy special care has been given to the structure, the accessability and ease of modification. The data base is developed primarily for use on Personal Computers (PC s). The PC enhances accessability due to their widespread use. The final version of the data base will have the format of a tree-structure, which will allow the user to access the information in form of raw data, plots or equations. In many instances the equations are not simple fits to the data but we have developed phenomenological equations based on analytical interpretations.

In section 2 we will list materials and properties investigated for this blanket study. Section 3 is a list of phenomenological equations and mathematical fits for all materials and properties considered. Section 4 describes our efforts to develop a swelling equation based on the few 
experimental data points avallable for breeder materials. In section 5 we. investigate the sintering phenomena for ceramics.

\section{Material Properties}

The present reactor study investigated the following materials as candidates for the Fusion Power Core (FPC): ferritic steels HT-9, and $21 / 4$ $\mathrm{Cr}-1$ Mo, blanket breeder ceramies $\mathrm{LiAlO}_{2}$ and $\mathrm{Li}_{2} \mathrm{D}$; and blanket neutron muitiplier material beryllium. $\mathrm{Li}_{2}{ }^{0}$ was ruled out as a candidate for solid breeder material due to its high anticipated swelling behavior. Nevertheless we will discuss some of our findings about $\mathrm{Li}_{2} \mathrm{O}$ in Section 4.

The data on all materials was compiled form various sources, mainly the Materials Handbook for Fusion Energy Systems [1], and recent experimental investigation by Hollenberg [2] and Rasneur [3]. The Materials Handbook includes a comprehensive work on the ferritic steel $21 / 4 \mathrm{Cr}$. $1 \mathrm{Mo}$, of which much information has been detemined over the last decade. Mechanical properties data and thermal data most relevant reactor blanket designs are listed in Table 1 . Some of the data on the ferritic steel HT-9 is also derived from the Materials handbook. Most of the data used for this report, however, comes directly from the Sandvik Steel Co. reports on HT-9 mechanical properties [4].

Other data on the ferritic steels include sceady state chermal creep correlations [5]. The expression used for swelling of the ferricic steel $21 / 4 \mathrm{Cr}-1 \mathrm{Mo}$ is from work by Gelles and Pulgh (6) on irradiation induced swelling. Since observed swelling is a bilinear phenomenon, this fit to the data produces a smooth transition from one swelling rate to another at the critical value of dose. The expression used for the ferritic steel HT-9 
is based on a ftt fron work by Ghonten [7]. It does not produce a smaoth transition, and therefore the value of swolling should be set to zero below the incubation dose. The correlation used for trradiation creep of HT-9 is also based on an expression previously developed for $21 / 4 \mathrm{Cr}$ - I Mo [6].

Information on material properties of 11 thium aluminate and lithium oxide is derived chiefly from Hollenberg [2] and Rasneur's work [3]. Some of the data displayed in this paper is in kaw format, and requires transforming into suitable units for proper use. In addition, much of the information was sparse and scattered, and extrapolation techniques were used to fill in the data. In particular, the phenomenon of swelling and process of sintering of these breeder ceramics have not been measured in much detail. However, correlations were developed from these data and are discussed in section 3.

In order to determine the tritium breeding behavior of $\mathrm{LiAlO}_{2}$, Rasneur [3] examined propertles of very homogeneous microtextures of porous $\mathrm{LiAlO}_{2}$ in an In-sicu tritium extraction experiment. From a large field of homogeneous microstructures, empirical equations were deduced as a function of porosity, grain dianeter and tempetature. Mechanical and thermal propertles examined include: ultra sound velocity, Young's modulus, ultimate compressive strength, creep and thermal shock. Physico-chemical propertles such as electrical resistivity and sensitivity to alr were also investigated. Raseneur found porous $\mathrm{LLAlO}_{2}$ to be an insulator even up to $600^{\circ} \mathrm{C}$. Exposed to air, LiAlO 2 will react with $\mathrm{H}_{2} \mathrm{O}$ vapor to form $\mathrm{LiOH}$ molecules. LiOH will then react with $\mathrm{CO}_{2}$ to ferm $\mathrm{Li}_{2} \mathrm{CO}_{3}$ compounds. Rasseneur's experiments showed that porous lithium aluminate exposed to air w11 have about 10 monolayers corroded after one year. 1.e. less than 18 for an average grain size of $0.4 \mu \mathrm{m}$ in diameter. In water however, the reaction on tdentical samples was total after about only three months. 
3. Phenomenological and Emplrical Equations.

This section lists the data base equations which are the results of our phenomenological investigations and purely empirfcal fits performed by us or by mentioned authors.

We start with ferritic steels HT-9 and $21 / 4 \mathrm{Cr}-1$ Mo and conclude this collection with breeder material properties listed in Table $I$.

TABLE 1

Summary for Blanket Material Properties

Current Status $(12 / 85)$

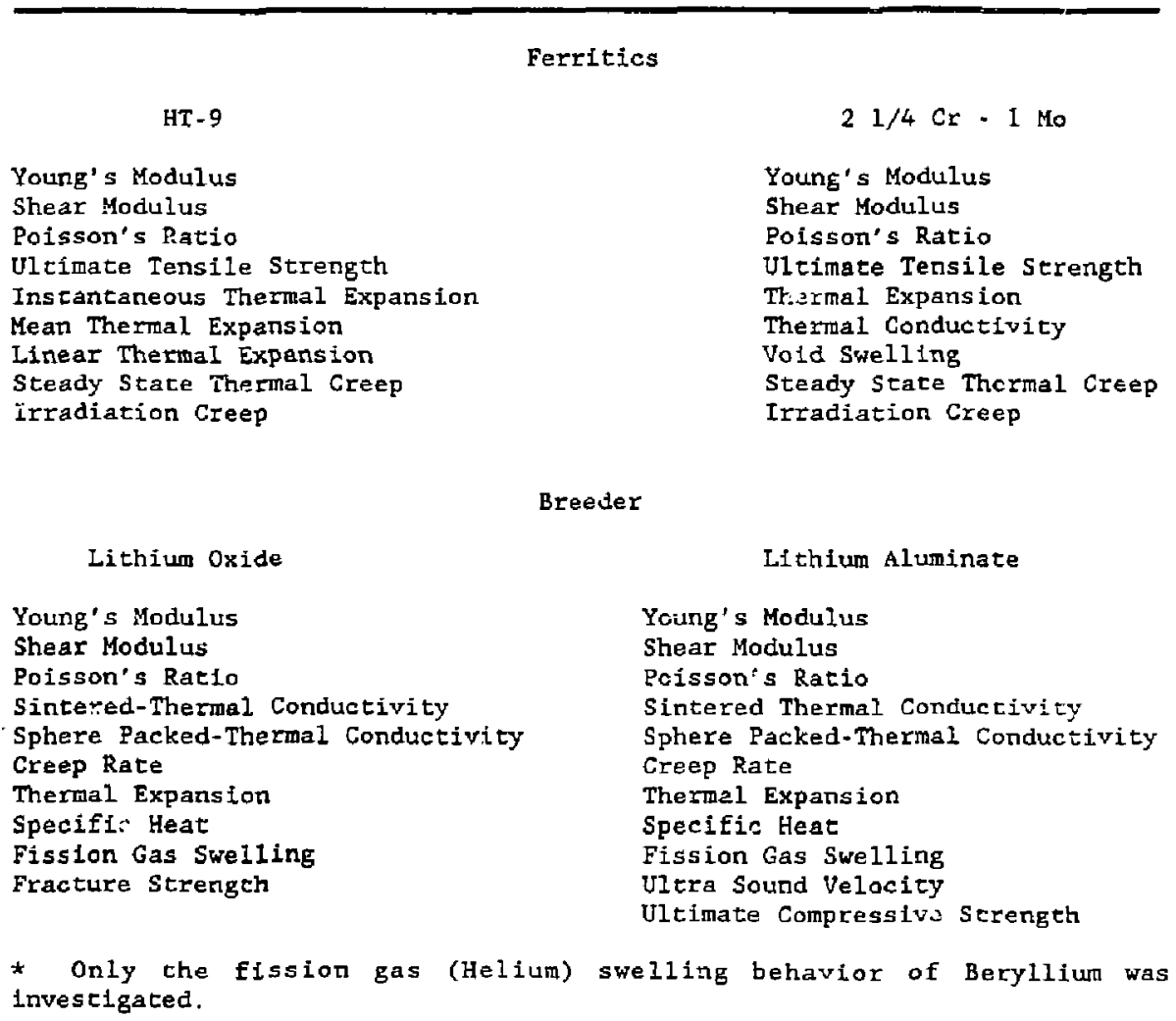




\section{I FERRITIC STEELS}

1. HI-9

$$
\begin{aligned}
& \text { Young's Modulus } \mathrm{MM}\left(\mathrm{T}^{\circ} \mathrm{F}\right) \mathrm{MPa} \\
& \mathrm{MM}-\left(\mathrm{A}_{1}+\mathrm{A}_{2} \mathrm{~T}+\mathrm{A}_{3} \mathrm{~T}^{2}\right) \times 10^{3} \\
& \text { Poisson's Ratio } \quad \mathrm{PR}\left(\mathrm{T}^{\circ} \mathrm{F}\right) \\
& \text { PR }=\mathrm{B}_{1}+\mathrm{B}_{2} \mathrm{~T}+\mathrm{B}_{3} \mathrm{~T}^{2}
\end{aligned}
$$

Shear Modulus $S M\left(T^{\circ}{ }^{\circ}\right)$ MPa

$$
\begin{aligned}
& \text { SM - MM/2(I+PR) } \\
& \text { Ultimate Tensile Strength }\left[8^{1} \quad \text { UTS ( } \mathrm{K}\right) \quad \mathrm{MPa} \\
& 473<\mathrm{T}<873 \\
& \text { UTS }-\mathrm{C}_{1}+\mathrm{C}_{2} \mathrm{~T}+\mathrm{C}_{3} \mathrm{~T}^{2}+\mathrm{C}_{4} \mathrm{~T}^{3}
\end{aligned}
$$

Instantarzous Thermal Expansion Goefficient ITE (T ${ }^{\circ} \mathrm{C}$ ) $10^{-6},{ }^{\circ} \mathrm{C}$

$$
\begin{aligned}
2 i & =T<650 \\
\text { ITE }=D_{1}+D_{2} T & +D_{3} T^{2}
\end{aligned}
$$

Mean Thermal Expansion Coefficient MTE $\left(t^{\circ} \mathrm{C}\right) 10^{-6} /{ }^{\circ} \mathrm{C}$

$$
\begin{gathered}
37<T<650 \\
\operatorname{MTE}-\left(E_{1}+E_{2} T+E_{3} T^{2}+E_{4} T^{3}\right) /(T-25)
\end{gathered}
$$


L.Inear Thermal Expansion LTE $\left(T^{\circ} \mathrm{C}\right)$ m

$$
\begin{gathered}
21<T<650 \\
\text { ITE }-F_{1}+F_{2} T+F_{3} T^{2}+F_{4} T^{3}
\end{gathered}
$$

Steady State Thermal Creep Rate [8] TCR (o ksi, I K) $\mathrm{s}^{-1}$

$$
\begin{aligned}
\mathrm{TCR}=\left(\mathrm{B}^{\prime} / \mathrm{kT}\right)\left(\sigma-\sigma_{1_{0}}\right)^{3} \mathrm{e}(-\mathrm{Q} 1 / \mathrm{kT}\rangle \\
\mathrm{B}^{\prime}-7.385 \times 10^{-3} \\
\sigma_{1_{0}}=\mathrm{aT}+\mathrm{C} \mathrm{\textrm {ki }} \\
\mathrm{a}=-.2185 \mathrm{ksi} / \mathrm{K} \\
\mathrm{C}=198.1783 \\
\mathrm{Q} 1-1.23 \mathrm{eV}
\end{aligned}
$$

Irradiation Creep [y] IC ( $\sigma_{\text {eff }} \mathrm{ksi}, \Phi \mathrm{n} / \mathrm{cm}^{2}-s, t s$ ) $q$

$$
\begin{aligned}
& I C=K^{\prime} \sigma_{\text {eff }}^{1.5} \Phi \tau \\
& \quad X^{\prime}=0.4 \times 10^{-4}\left(\mathrm{MPa}^{-1}\left(10^{22} \mathrm{n} / \mathrm{cm}^{2}\right)\right.
\end{aligned}
$$

Void swelling [ 8$]$ VS $\left(\delta\right.$ DPA, $\left.T{ }^{\circ} \mathrm{C}\right)$

$$
\begin{aligned}
\text { VS }= & c^{\prime}\left(\delta-\delta_{0}\right) \mathrm{e}^{-((\mathrm{T}-425) / 59)^{2}} \\
c^{\prime} & =0.025 \quad / \mathrm{DPA} \\
\delta_{0} & =90 \mathrm{DPA}
\end{aligned}
$$


2. $21 / 4 \mathrm{Cr}-1 \mathrm{MO}$

Young's Modulus YM ( $T$ ' $F$ ) MPa

$$
Y M=\left\langle G_{1}+G_{2} T+G_{3} T^{2}+G_{4} T^{3}\right\rangle \times G_{5}
$$

Foisson's Ratio $P R\left(T{ }^{\circ} F\right)$

$$
P R-H_{1}+H_{2} T+H_{3} T^{2}
$$

Shear Modulus SH (I $\left.{ }^{\circ} \mathrm{F}\right) \mathrm{MPa}$

$$
\mathrm{SM}=\mathrm{YM} / 2(1+\mathrm{PR})
$$

vitimate Tensile strength [8] UTS (T K) MPa

$$
473<\mathrm{T}<873
$$

$$
U T S-I_{1}+I_{2} T+I_{3} T^{2}+I_{4} T^{3}
$$

Thermal Exp:unsion Coefficient TE (T $\left.{ }^{\circ} \mathrm{C}\right) 10^{-6} /{ }^{\circ} \mathrm{C}$

$$
\begin{array}{r}
20<\mathrm{T}<700 \\
\mathrm{TE}-J_{1}+\mathrm{J}_{2} \mathrm{~T}+\mathrm{J}_{3} \mathrm{~T}^{2}
\end{array}
$$

Thermal Conductivity TC $\left(T{ }^{\circ} \mathrm{C}\right) \mathrm{W} / \mathrm{cm}-\mathrm{K}$

$$
\begin{gathered}
25<\mathrm{T}<700 \\
\mathrm{TC}-\mathrm{K}_{1}+\mathrm{K}_{2} \mathrm{~T}+\mathrm{K}_{3} / \mathrm{T}+\mathrm{K}_{4} \mathrm{~T}^{2}
\end{gathered}
$$


Steady State Thermal Creep Rate[8] TCR ( $\sigma \mathrm{ksi}, \mathrm{T} \mathrm{K}$ ) $\mathrm{s}^{-1}$

$$
\begin{aligned}
\operatorname{TCR}-\left(\mathrm{A}^{\prime} / \mathrm{kT}\right)\left(\sigma-\sigma_{2}\right)^{4} \mathrm{e}^{(-\mathrm{Q} 2 / \mathrm{kT})} \\
1 \mathrm{n} \mathrm{A}^{+}-17.2898 \\
\sigma_{2_{0}}=\mathrm{aT}+\mathrm{C} \\
\mathrm{a}=-0.0628 \\
C=51.7929 \\
\mathrm{Q}^{2}-2.767 \mathrm{eV}
\end{aligned}
$$

Irradiation Creep [9] IC ( $\sigma_{\text {eff }} \mathrm{ksi}, \Phi \mathrm{n} / \mathrm{cm}^{2}-\mathrm{s}, \mathrm{t} s$ ) $\%$

$$
\text { IC } \begin{aligned}
-\mathrm{K}^{\prime} & { }_{\sigma_{\mathrm{eff}}}^{1.5} \Phi \mathrm{t}+\mathrm{DS} \sigma_{\text {eff }} \\
\mathrm{K} & =0.4 \times 10^{-4}(\mathrm{MPa})^{-1}\left(10^{22} \mathrm{n} / \mathrm{cm}^{2}\right)(8) \\
\mathrm{D} & - \text { swelling enhanced creep coefficient } \\
& =2.7 \times 10^{-5}(\mathrm{MPa})^{-1}\left(10^{22} \mathrm{n} / \mathrm{cm}^{2}\right) \\
\mathrm{S} & =\text { fractional swelling rate }\left(\mathrm{g} / 10^{22} \mathrm{n} / \mathrm{cm}^{2}\right)
\end{aligned}
$$

Void Swelling [9] vs $\left(\Phi \mathrm{n} / \mathrm{cm}^{2}, t s\right)$ \%

$$
\begin{aligned}
v S=v S_{1} & -D \\
v s_{1} & =R\left[\Phi t+\frac{1}{\alpha} \ln \left[\frac{1 \cdot \exp (\alpha(y-\Phi t))}{1+\exp (\alpha t)}\right]\right] \\
D & =\text { Densification Term } \\
& =D(1-e(-\lambda \oint t)
\end{aligned}
$$




$$
\begin{aligned}
& \text { D - steady state density (- }-0.088) \\
& \lambda \text { - transition parameter }\left(-3\left(10^{22} \mathrm{n} / \mathrm{cm}^{2}\right)\right) \\
& \gamma-\text { swelling incubation parameter } \\
& -c_{1} \exp \left(c_{2}\left(T-c_{3}\right)^{2}\right) \\
& c_{1} \text { - transition } \$ \text { irameter } \\
& \mathrm{c}_{2}-2.0 \times 10^{23} \mathrm{n} / \mathrm{cm}^{2} \\
& c_{3}-5.0 \times 10^{-5}(\mathrm{~K})^{-2} \\
& a=\text { swelling rate }\left(-0.5\left(10^{22} \mathrm{n} . \mathrm{cm}^{2}\right)^{-1}\right) \\
& \mathrm{R}=0.258 / 10^{22} \mathrm{n} / \mathrm{cm}^{2}
\end{aligned}
$$


Table of Constants for Ferritic Steel Equation

1. HT-9

$$
\begin{aligned}
& \text { A1 : } 213.28 \\
& \text { A2 : }-4.799 \times 10^{-2} \\
& \text { A3 : }-4.065 \times 10^{-6}
\end{aligned}
$$

C1 : 299.7298

C2 : -1.11815

C3 : $1.98127 \times 10^{-3}$

C4: $-1.179135 \times 10^{-6}$

$E 1-\cdot 235.2$

E2 -9.2207

E3 - $7.5806 \times 10^{-3}$

$\mathrm{E} 4=-3.5412 \times 10^{-6}$
$B 1: 0.2762$

B2 : $B .9309 \times 10^{-5}$

B3 : $-6.262 \times 10^{-8}$

$D 1: 9.2207$

$\mathrm{D} 2: 1.5161 \times 10^{-2}$

D3 : $-1.0624 \times 10^{-5}$

F1 : -.23520

F2 $: 9.2207 \times 10^{-3}$

$73: 7.5806 \times 10^{6}$

F4: $-3.5412 \times 10^{-9}$ 


$$
\begin{aligned}
& G 1=31.1 \\
& G 2=-1.359 \times 10^{-2} \\
& G 3=2.505 \times 10^{-5} \\
& G 4=-2.007 \times 10^{-8} \\
& G 5=6.89475 \times 10^{3}
\end{aligned}
$$$$
\mathrm{HI}-0.254
$$$$
\mathrm{H} 2-1.54 \times 10^{-4}
$$$$
133=-2.26 \times 10^{-7}
$$

$11-152.7886$

$J 1-11.281$

I2 --0.5938

$J 2=€ .97817 \times 10^{-3}$

$13=1.43787 \times 10^{-3}$

$J 3--3.8036 \times 10^{-6}$

$14=-1.060566 \times 10^{-6}$

$\mathrm{K} 1-0.64986$

$\therefore 2=-0.2784 \times 10^{-3}$

$\mathrm{k} 3--82,462$

$K 4-6407.375$ 


\subsection{BREEDER CERAMICS}

I. Lithium Oxide (Li2 20$)$

$$
\begin{aligned}
& T_{\pi}=1433{ }^{\circ} \mathrm{C} \\
& \rho_{\text {Li }}=0.93 \mathrm{~g} / \mathrm{cm}^{3} \\
& k=3.4 \mathrm{~W} / \mathrm{mk}
\end{aligned}
$$

Notations are as follow:

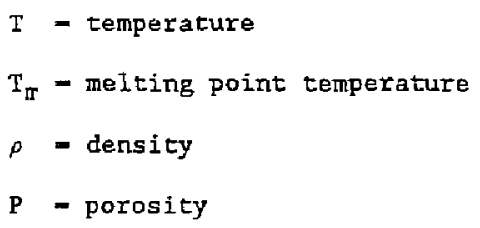

1) YOUNG'S MODULES (E)

$E=140 \exp (-4 \mathrm{P})\left[(1-\mathrm{T}) \exp \left(1-\mathrm{T}_{\mathrm{m}} / \mathrm{T}\right)\right] \mathrm{GPa}$

2) rOISSON'S RATIO ( $\eta$ )

$\eta=0.25$

3) SHEAR MODULUS (G)

$$
G=\frac{E}{2(I+\eta)} \quad(\mathrm{GPa})
$$

4) THERMAL EXPANSION COEFFICIEN'S $(\alpha)$

$$
\begin{aligned}
a=a & +1.72 \times 10^{-2} \mathrm{~T} \\
a & =16.05 \text { for } \mathrm{T} \text { in }{ }^{\circ} \mathrm{K} \\
a & =20.75 \text { for } \mathrm{T} \text { in }{ }^{\circ} \mathrm{C}
\end{aligned}
$$


5) FRACTURE STRENGTH ( $\left.\sigma_{\mathbf{f}}\right)$

$$
\begin{aligned}
& \sigma_{E}=\sigma_{0}[1-44 \exp (-7000 / \mathrm{T})] \quad(\mathrm{MPa}) \\
& \sigma_{0}-108 \mathrm{~d}^{-0.4} \exp (-10 \mathrm{P})(\mathrm{MPa}) \\
& \mathrm{d}=\text { grain diameter } \\
& \mathrm{T}=\text { in }{ }^{\circ} \mathrm{K}
\end{aligned}
$$

6) THERKAL CONDUCTIVITY (k)

\section{SPHERE PACKED (a 87\% SPF)}

$400^{\circ} \leq \mathrm{T} \leq 1100^{\circ} \mathrm{K}$

A) Unirradiated

(i) Helium fill-gas pressure -6 atm.

$$
k=14.220-2.076 \ln (T)+0.3506(\ln T)^{2} \mathrm{~W} / \mathrm{mK}
$$

(ii) Helium fill-gas pressure - 2 atm.

$k=21.683-4.669 \operatorname{lil}(\mathrm{T})+0.259(\operatorname{ln~T})^{2} \mathrm{~W} / \pi \mathrm{KK}$

(iii) Heifum fill-gas pressure - I atm.

$$
k=15.594-3.295 \ln (T)+0.182(\ln T)^{2} \mathrm{~W} / \mathrm{mK}
$$

B) Irradiated ( $\left.\Phi \mathrm{t}>0.5-2 \times 10^{26} \mathrm{n} / \mathrm{m}^{2}\right)$

i) Helium fill-gas pressure -6 atm.

$$
k--0.612+1.6791 \mathrm{n}(\mathrm{T}) \cdot 0.1905(1 \mathrm{nT})^{2} \mathrm{w} / \mathrm{mk}
$$


ii) Hellum fill-gas pressure - 2 atm.

$k=8.937-1.3392 \pi(T)+0.00387(2 \pi T)^{2} \quad W / \mathrm{mK}$

iii) Helium fill-gas pressure - $1 \mathrm{acm}$.

$k-16.035-3.681 \mathrm{In}(\mathrm{T})+0.223(1 \mathrm{nT})^{2} \quad \mathrm{\omega} / \mathrm{mK}$

SINTERED

$400^{\circ} \leqslant T \leq 1100^{\circ} \mathrm{K}$

A) Unirradiated

1) $\mathrm{TD}=1008$

$k=42.011-5.4071 n(T) \quad W / m K$

ii) TD $=84$ i

$k-72.404-16.1461 n(T)+0.899(\operatorname{InT})^{2} W / \mathrm{mK}$

i1i) TD $=70 \%$

$k-49.701-11.724 \ln (T)+0.708(\operatorname{lnT})^{2} \quad \mathrm{H} / \mathrm{mK}$

B) Irradiated ( $\$ \mathrm{t}>0.5-2 \times 10^{26} \mathrm{n} / \mathrm{m}^{2}$ )

1) $\mathrm{TD}=85 \%$

$k-45.602-11.532 \ln (T)+0.76(\operatorname{lnT})^{2} \mathrm{~W} / \mathrm{aK}$ 


$$
\text { (1) } \begin{aligned}
\text { TD } & =70 \mathrm{~V} \\
\mathrm{k} & =29.353-7.75 \ln (\mathrm{T})+0.545(\mathrm{lnT}) \mathrm{H} / \mathrm{mK}
\end{aligned}
$$

7) CREEP RATE

$$
\epsilon=A \sigma \exp \left(-E_{c} / k I\right)\left(h r^{-I}\right)
$$

It is assumed that $\mathrm{Li}_{2} \mathrm{O}$ will follow approximately the creep Fate of $\mathrm{UO}_{2}$. Constants for the creep equations have been correlated using melting point temperatures.

$$
\begin{aligned}
A= & 140 \\
a- & 1.49 \times 10^{4}\left(\mathrm{kN} / \mathrm{m}^{2}\right) \\
E_{c}- & 2.03(\mathrm{eV}) \\
& \text { for } 570^{\circ} \leq I \leq 900^{\circ} \mathrm{C}
\end{aligned}
$$

8) SPECIFIC HEAT (cp)

$$
\begin{aligned}
& \alpha-A_{1}+A_{2} T+A_{3} / T^{2} \mathrm{~J} / \mathrm{kgK} \\
& A_{1}-2.5179 \times 10^{3} \\
& A_{2}=0.3328 \\
& A_{3}-8.382 \times 10-7 \\
& \quad \text { for } 306 \leq \mathrm{T} \leq 1073^{\circ} \mathrm{K}
\end{aligned}
$$

9) SWELLINE ( $\Delta V / N)$

$$
\frac{\Delta y}{V}-8.87 \times 10^{-30}\left[\frac{\operatorname{TfGL}}{\mathrm{N}^{1 / 3}}\right]^{3 / 2}
$$




$$
\begin{aligned}
& f \text { - fraction of Hellum retained } \\
& \text { G - Helium production rate }\left(\mathrm{cm}^{-3} \mathrm{sec}^{-1}\right. \text { ) } \\
& \text { E - time (sec) } \\
& \text { N - Bubble number density }\left(\mathrm{cmn}^{-3}\right)
\end{aligned}
$$

\section{I. I.ithium Aluminate}

$$
\begin{aligned}
& \mathrm{T}_{\mathrm{m}}=1610^{\circ} \mathrm{C} \\
& \rho_{\mathrm{Li}}=0.28 \mathrm{~g} / \mathrm{cm}^{3} \\
& \mathrm{k}=1.6 \mathrm{~W} / \mathrm{mK}
\end{aligned}
$$

1) UITRA SOUND (1MHz) VELOCITY (c)

$$
\begin{gathered}
c-c_{0}\left(1-\frac{P}{0.7}\right)\left(\mathrm{km}^{-3}\right. \\
\text { where: } c_{0}=8.4 \mathrm{kms}^{-1} \\
P=\text { Porosity }
\end{gathered}
$$

Temperacure dependence:

$$
\text { c is slowly decreasing by } 3 \text { to } 48 \text { form } \mathrm{c}-25^{\circ} \mathrm{C} \text { to } 700^{\circ} \mathrm{C} \text {. }
$$

2) YOUNG'S MODULUS (E)

$$
E=\rho c^{2}
$$

$$
\begin{aligned}
& \text { where } \rho \text { - apparent density } \\
& c \text { - uicra sound velocicy } \\
& E-E_{0}(I-P)\left(1-\frac{P}{0}\right)^{2} \text { (GPa) } \\
& \text { where } E_{0}-\rho_{0} c_{0}^{2}(\mathrm{GPa}) \\
& \text { for } \rho_{0}=2.615 \mathrm{~g} / \mathrm{cm}^{3}
\end{aligned}
$$




$$
\begin{gathered}
\vec{E}_{0}-184.5(\mathrm{GPa}) \\
E-184.5(1-\mathrm{P})\left(1-\frac{\mathrm{P}}{0.7}\right)^{2} \text { (GPa) }
\end{gathered}
$$

3) POISSON'S RATIO

$$
\begin{aligned}
& \eta=\eta_{0} /(1+0.1 P) \\
& \eta_{0}=0.25
\end{aligned}
$$

4) ULTIMATE COMPRESSIVE STREHGTH V.C.S.

$$
\begin{aligned}
& \text { U.C.S }-\frac{2\lfloor\exp (-10 P)]}{d \mathrm{~d}}(\mathrm{GPa}) \\
& \text { where: } d \text { - diameter of } \mathrm{LAAlO}_{2} \text { grain (in microns) } \\
& \text { For: } \quad d=0.42 \mu \\
& P=0.25 \\
& \text { the temperature }\left({ }^{\circ} \mathrm{K}\right) \text { dependence was found to be } \\
& \text { U.c.s. }=\frac{\exp (-20 \mathrm{P})}{\sqrt{\mathrm{d}}} \ln \left[\frac{2200}{\mathrm{~T}}\right] \quad(\mathrm{GPa} \text { ) } \\
& \text { valid for } 293^{\circ}<\mathrm{T}<1273^{\circ} \mathrm{K}
\end{aligned}
$$

5) THERMAL CREEP

Creep experiments were carried out at $700^{\circ} \mathrm{C}$ under three loads 50, 80 and $100 \mathrm{MPa}$ or samples with 0.25 porosity and $0.42 \mu$ grain diameter.

i) Under $100 \mathrm{MPa}$

$$
\frac{\text { Al }}{L}=-0.0135 \ln \frac{t}{72} \quad 200<t<1000 \mathrm{~h}
$$

1i) Under $80 \mathrm{MPa}$

$$
\frac{\mathrm{AL}}{\mathrm{L}}=-0.0045 \ln \frac{\mathrm{t}}{29} \quad 300<\mathrm{t}<2000 \mathrm{~h}
$$


111) Under $50 \mathrm{MPa}$

$$
\begin{aligned}
& \frac{\Delta \mathrm{L}-}{\mathrm{L}}-0.00143 \ln \frac{\mathrm{t}}{150} \quad 300<\mathrm{t}<2000 \mathrm{~h} \\
& \text { Extrapolation of (iii) to } 20 \text { years lesits to a creep of } 1 \mathrm{z} \\
& \text { at } 700^{\circ} \mathrm{C} .
\end{aligned}
$$

6) THERMAL CONDUCTIVITY (k)

$$
\begin{aligned}
& \text { SPHERE PACKED }(\approx 878 \text { SPF). } \\
& 500^{\circ} \mathrm{K} \leq \mathrm{T} \leq 1200^{\circ} \mathrm{K}
\end{aligned}
$$

A) Unfrradiated

1) Helium f111-gas rressure - 6 atm.

$$
k-25.670-5.5181 \mathrm{n}(\mathrm{T})+0.297(\mathrm{InT})^{2}(\mathrm{~W} / \mathrm{Mk})
$$

1i) Helium fill-gas pressure -2 atm.

$$
k-8.806-1.075 \ln (\mathrm{T}) \quad(\mathrm{H} / \mathrm{mK})
$$

1i1) Helium fill-gas pressure - 1 atm.

$$
k-6.897-0.827 \ln (\mathrm{T}) \quad(\mathrm{W} / \mathrm{mK})
$$

B) Irradiated $\left(\Phi t>0.5-2 \times 10^{26} \mathrm{n} / \mathrm{m}^{2}\right)$ 
1) Helfum fill-gis pressuze - 6 atm.

$$
k=8.385-0.9991 n(T) \quad(W / m K)
$$

if) Hellum fill-gas pressure 2 atrl.

$$
k=6.823-0.803 \ln (\mathrm{T}) \quad(\mathrm{W} / \mathrm{mK})
$$

iii) Helium fill-gas presaure - 1 atm.

$$
k-5.387-0.6191 \pi(T)(\omega / \mathrm{mK})
$$

\title{
SINTERED
}

$500^{\circ} \leq \mathrm{T} \leq 1200^{\circ} \mathrm{K}$

\begin{abstract}
A) Unirradiated
\end{abstract}
i) TD -1008

$$
k=21.01-2.651 \mathrm{n}(\mathrm{T}) \quad(\mathrm{W} / \mathrm{mK})
$$

11) $\mathrm{TD}-848$

$$
k=14.43=1.811 n(T) \quad(\omega / m K)
$$


iif) TD -708

$$
k=983-1.221 n(T) \quad(W / n K)
$$

B) Irradiated ( $\$ t=0.5 \cdot 2 \times 10^{26}$ )

1) TD -848

$$
k=11.097-1.373 \ln (T)(W / m K)
$$

ii) $\mathrm{TD}-70 \mathrm{~s}$

$$
k=6.45-0.771 n(T) \quad(W / m k)
$$

6) SEECIFIC HEAT ( $\left.c_{p}\right)$

The following measurements are available for a porosity of 0.22 and $d-0.43 \&$ grain diameter

\begin{tabular}{c|c} 
Temperature $\left(^{\circ} \mathrm{C}\right)$ & $\mathrm{c}_{\mathrm{p}-\left(\mathrm{caI} / \mathrm{g}^{\circ} \mathrm{G}\right)}$ \\
400 & 0.330 \\
500 & 0.348 \\
600 & 0.365
\end{tabular}

7) LINEAR EXPANSTON FACTOR ( $(\mathrm{L} / \mathrm{L})$

(In ${ }^{\circ} \mathrm{C}$ ) 


$$
\frac{\Delta L^{\prime}}{L^{\prime}}-7.43-2.95 \ln (T)+0.295(\ln T)^{2}
$$

\section{8) SWELIIKG $(\Delta V / v)$}

$$
\begin{aligned}
\frac{\Delta V}{V}-8.87 \times 10^{-30}\left[\frac{\mathrm{IfGE}}{\mathrm{N}^{2 / 3}}\right]^{3 / 2} \\
\text { where: } \quad \mathrm{T}-\text { temperature }\left({ }^{\circ} \mathrm{K}\right) \\
\mathrm{G}-\text { Helium production rate }\left(\mathrm{cm}^{-3} \mathrm{sec}^{-1}\right) \\
\mathrm{N}-\text { Bubble number density }\left(\mathrm{cm}^{-3}\right) \\
\\
\text { E }- \text { Fraction of helium retained }
\end{aligned}
$$


3.3 Selected Plots for the following Material Properties

HT-9 and $21 / 4 \mathrm{Cr}-1 \mathrm{Mo}$ :

Young's Modulus

Shear Modulus

Poisson's Ratio

Jltimat: 3 Tensile Strength

$\underline{\mathrm{Li}}_{2} \underline{\mathrm{O}}$ and $\underline{\mathrm{LiAlO}}_{2}$ :

Thermal Conductivity

Sintered Irradiaced 848,708 TD

Sintered Unirradiated 1098, 84t, 708 ID

Thermal Expansion Coefficient

Specific Heat

Creep Rate 

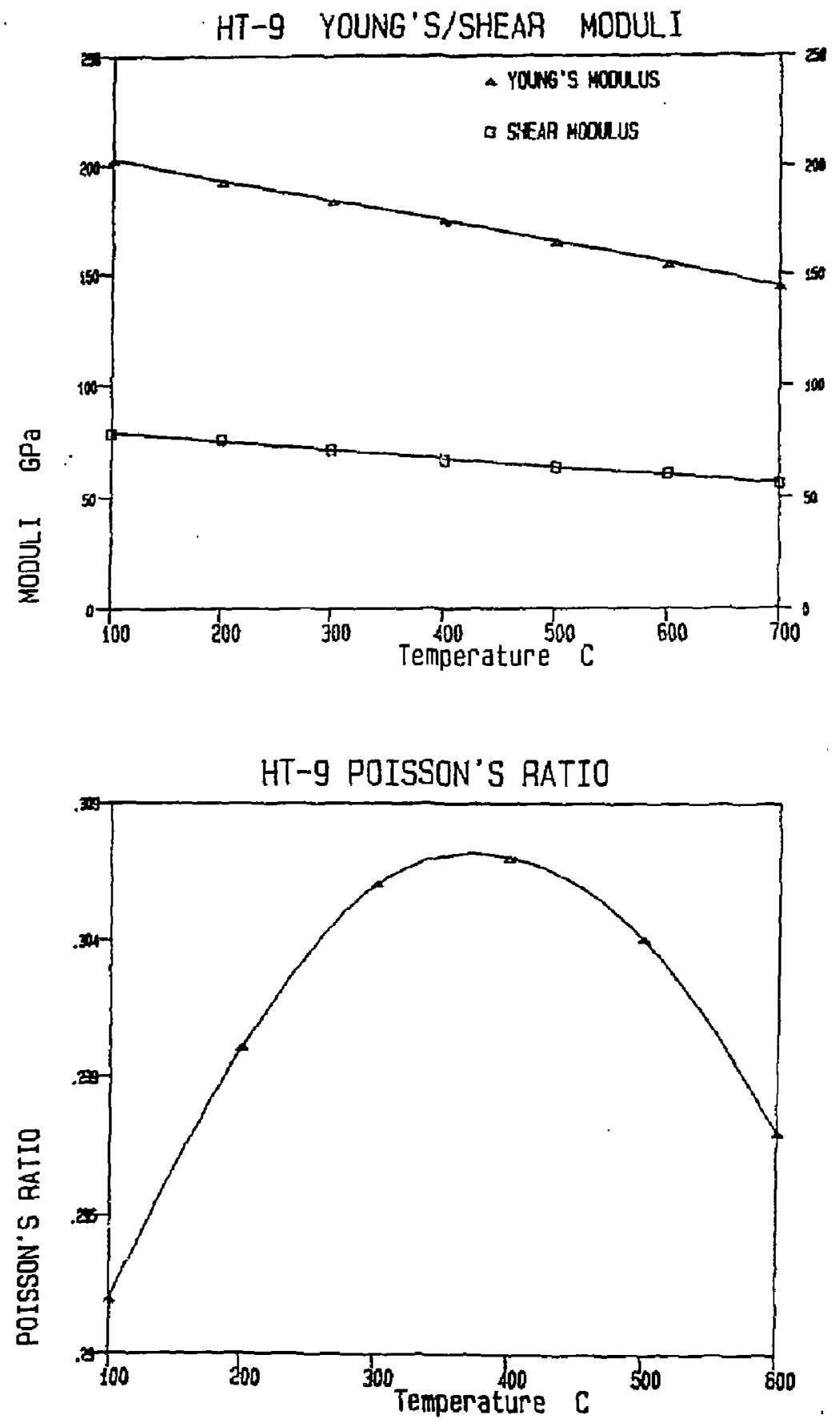


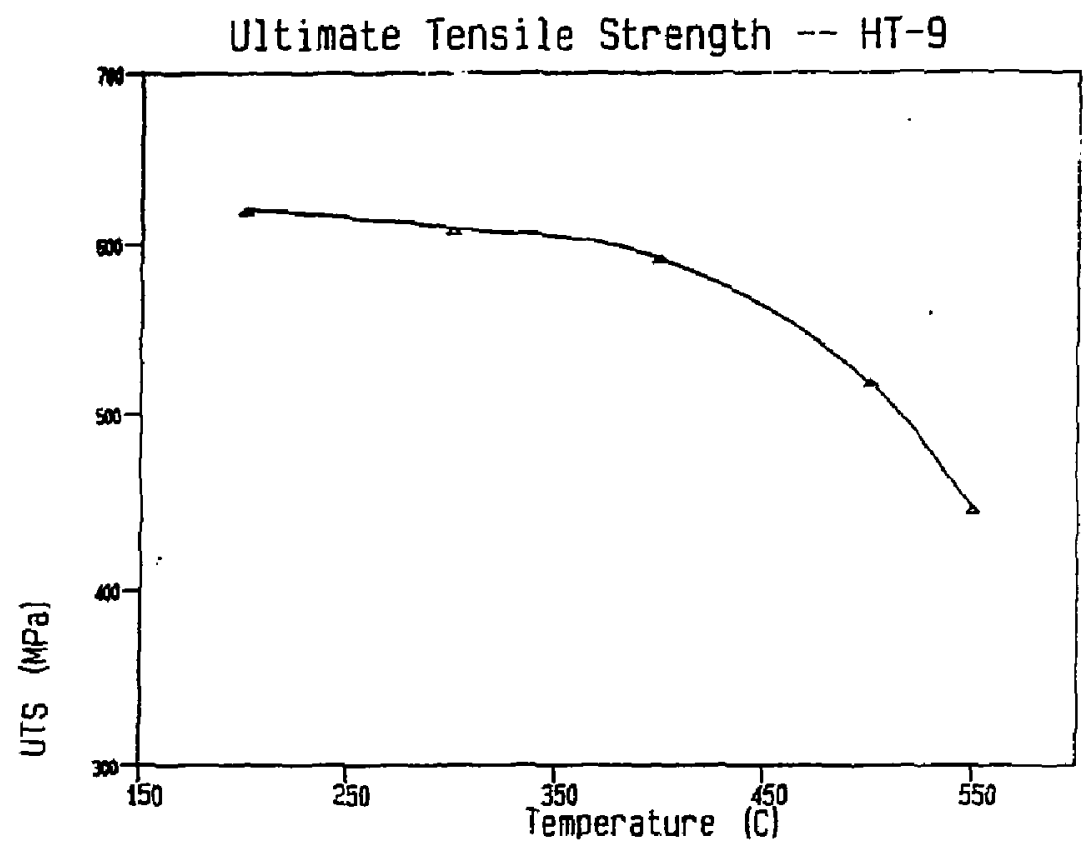



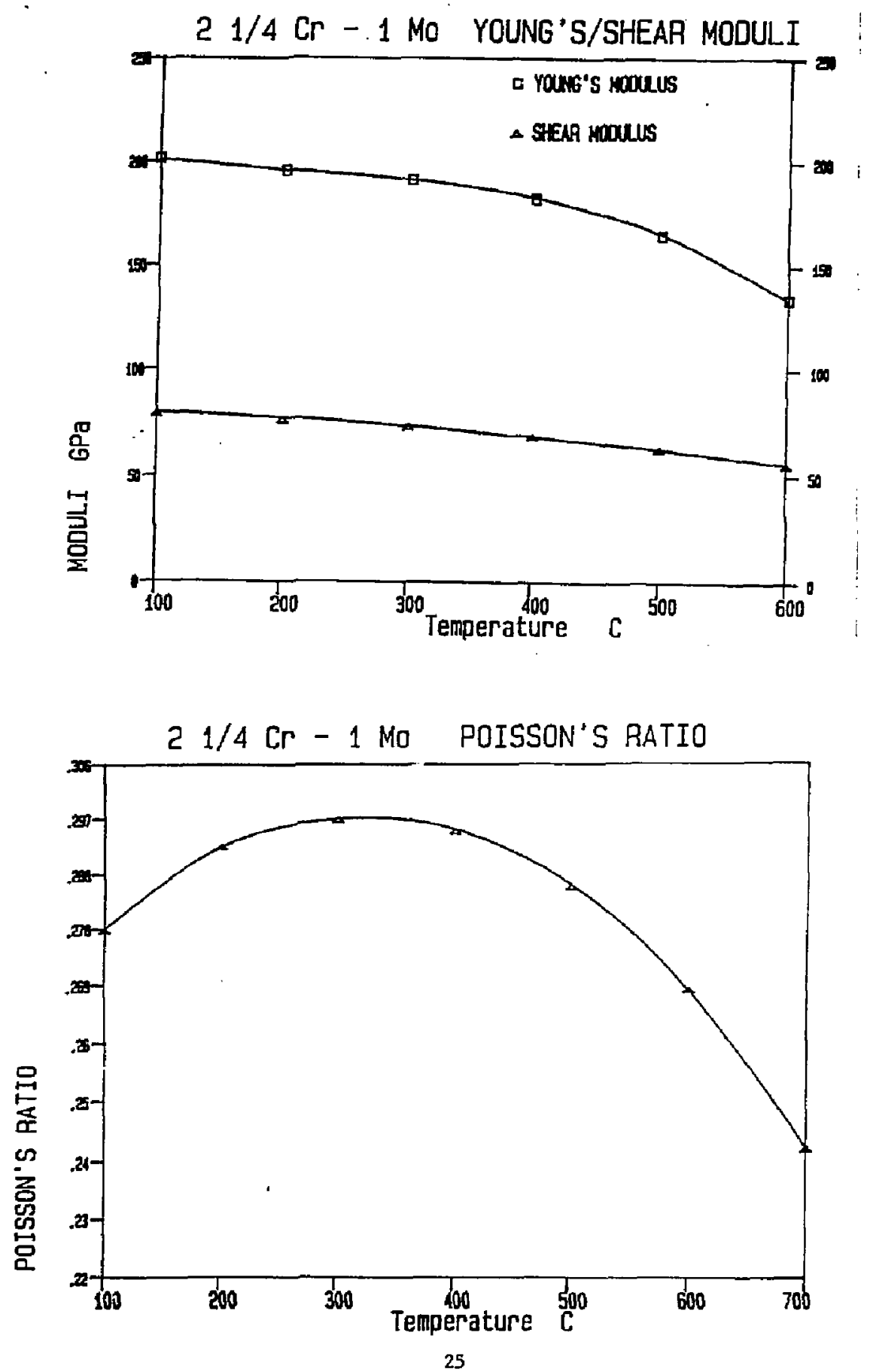


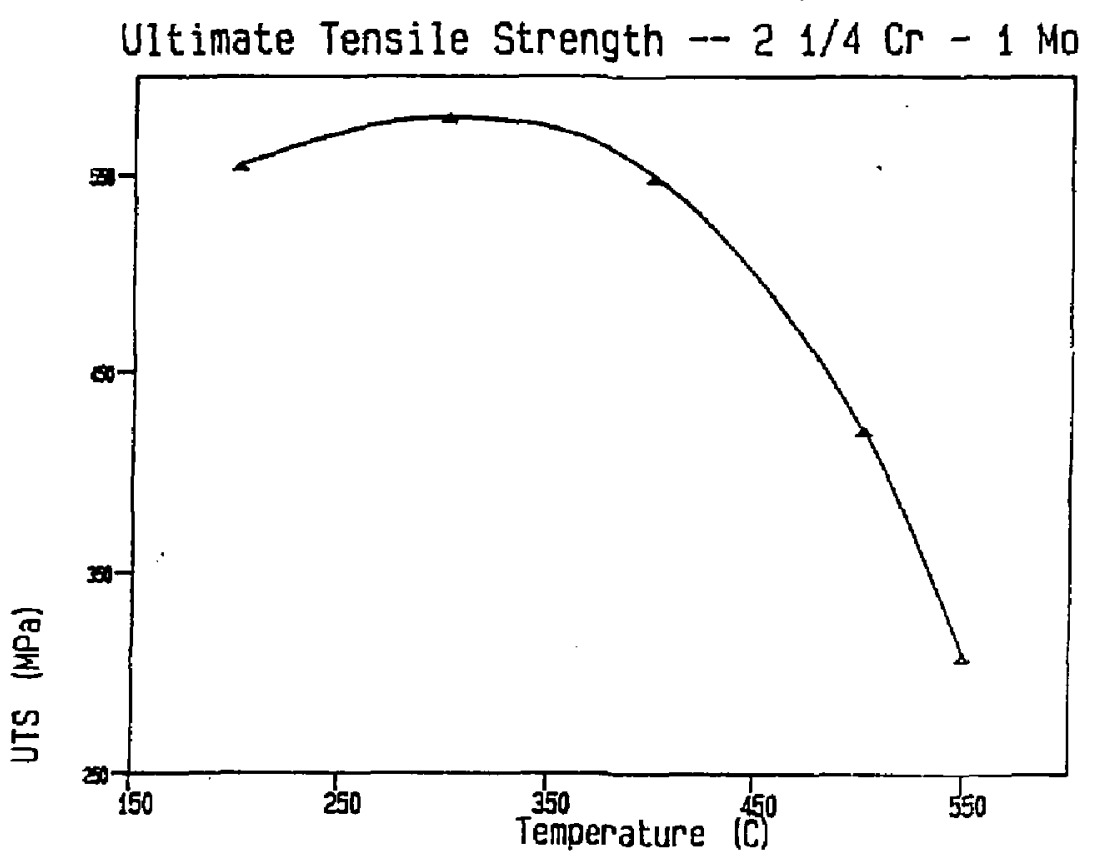



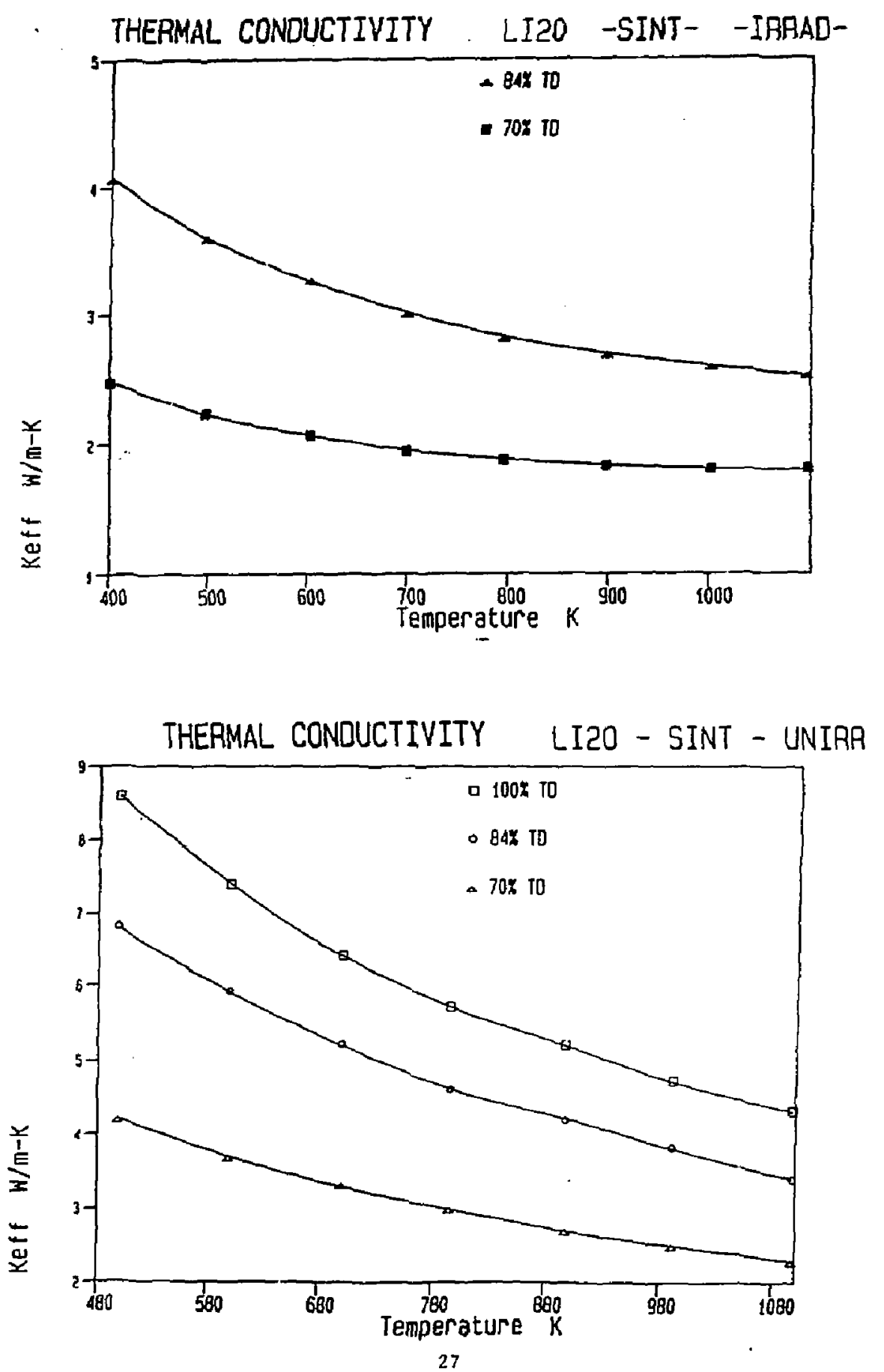

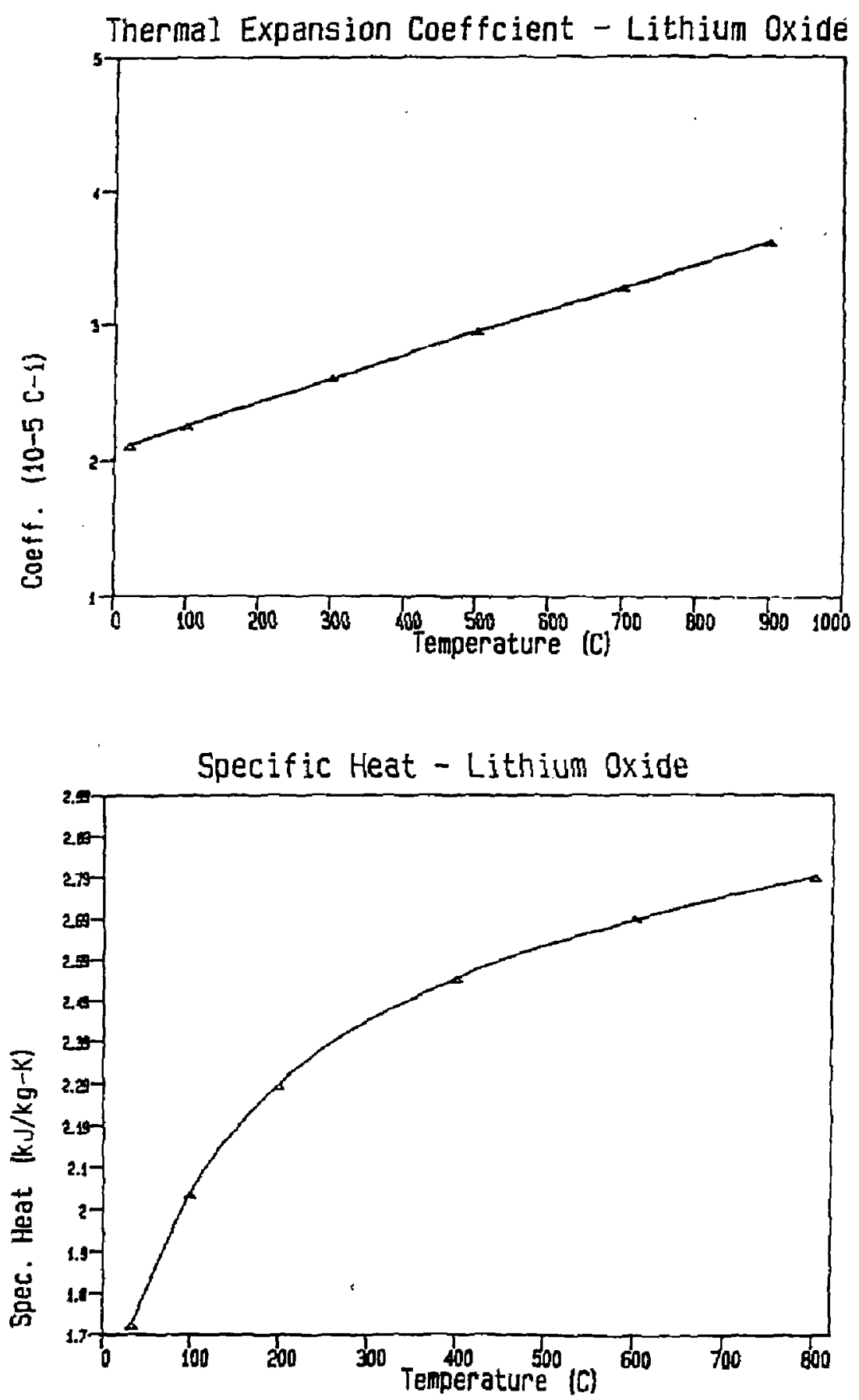


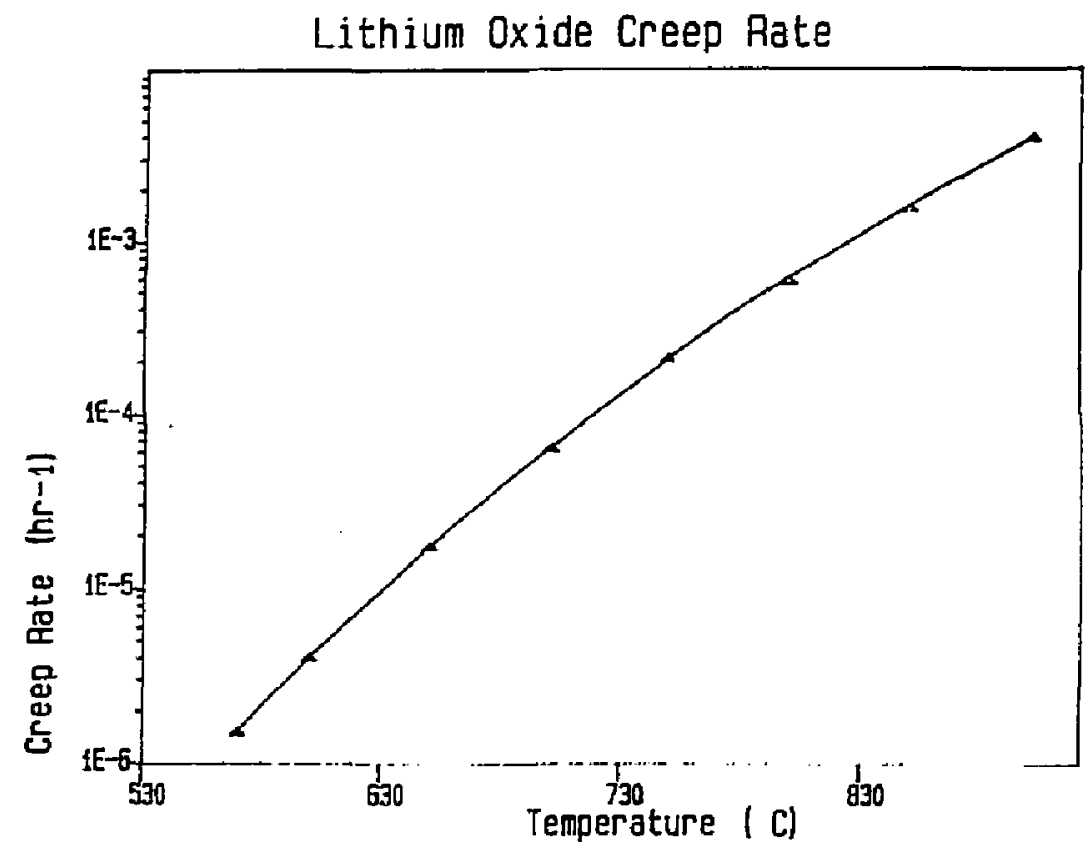



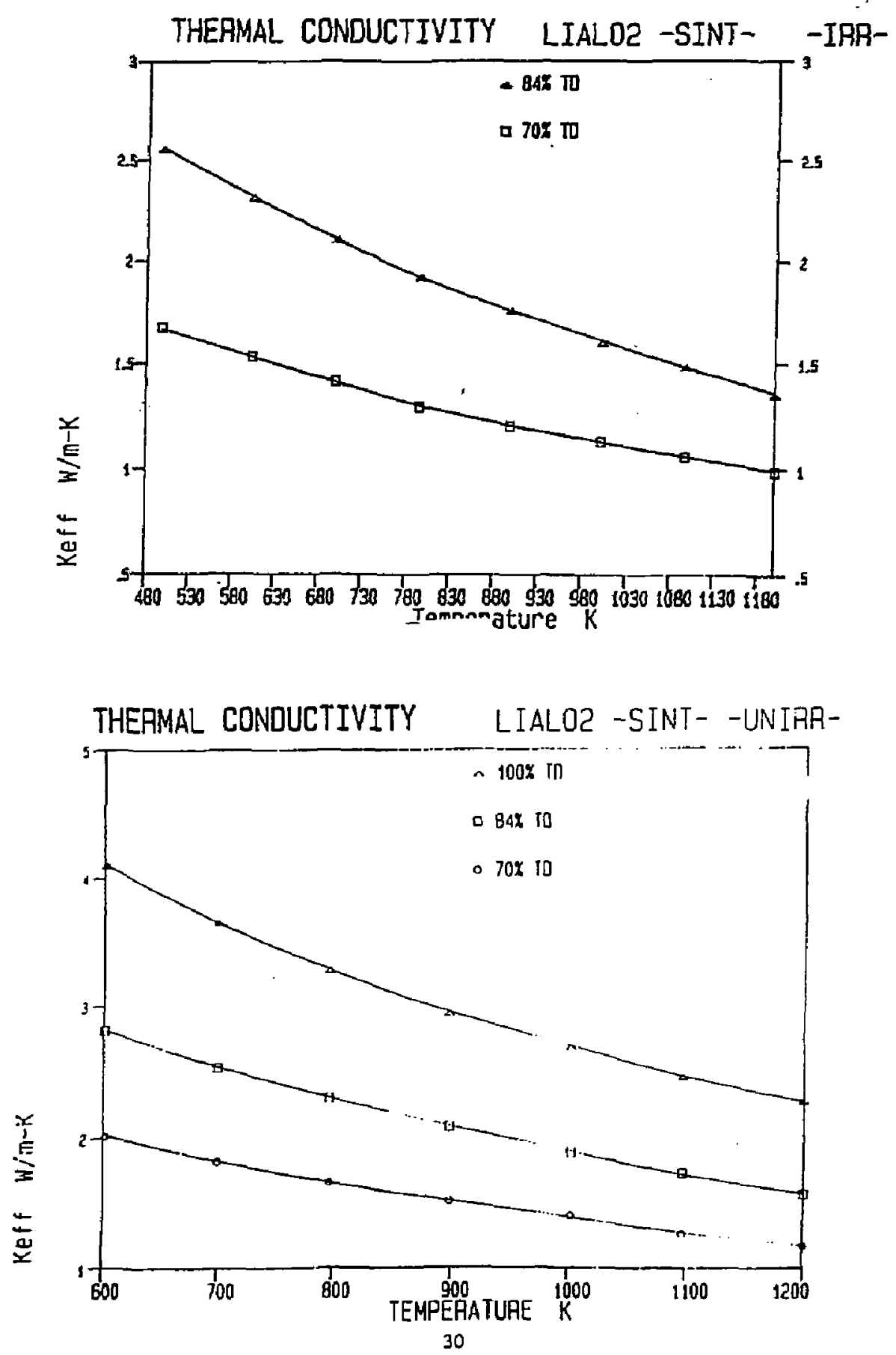

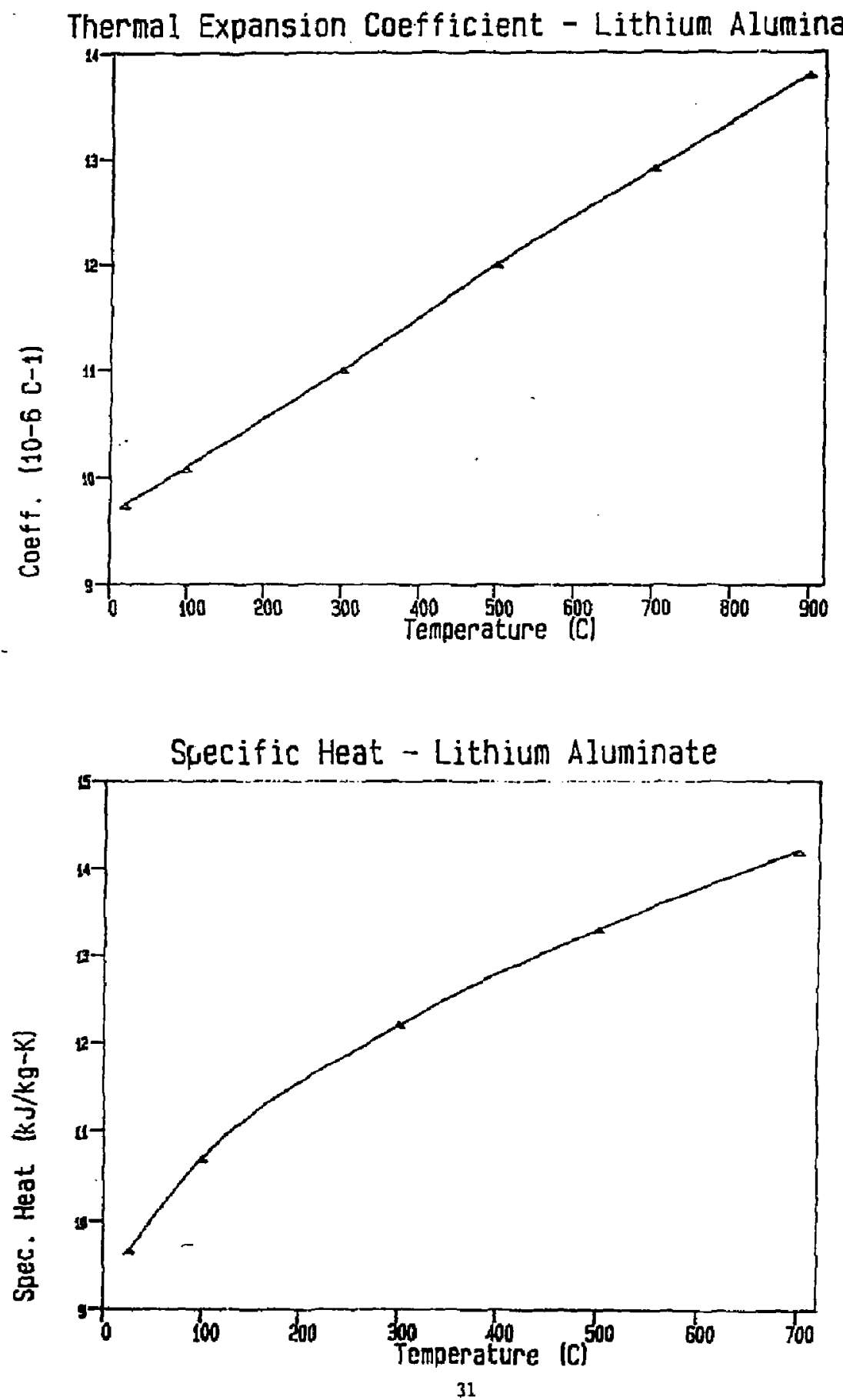


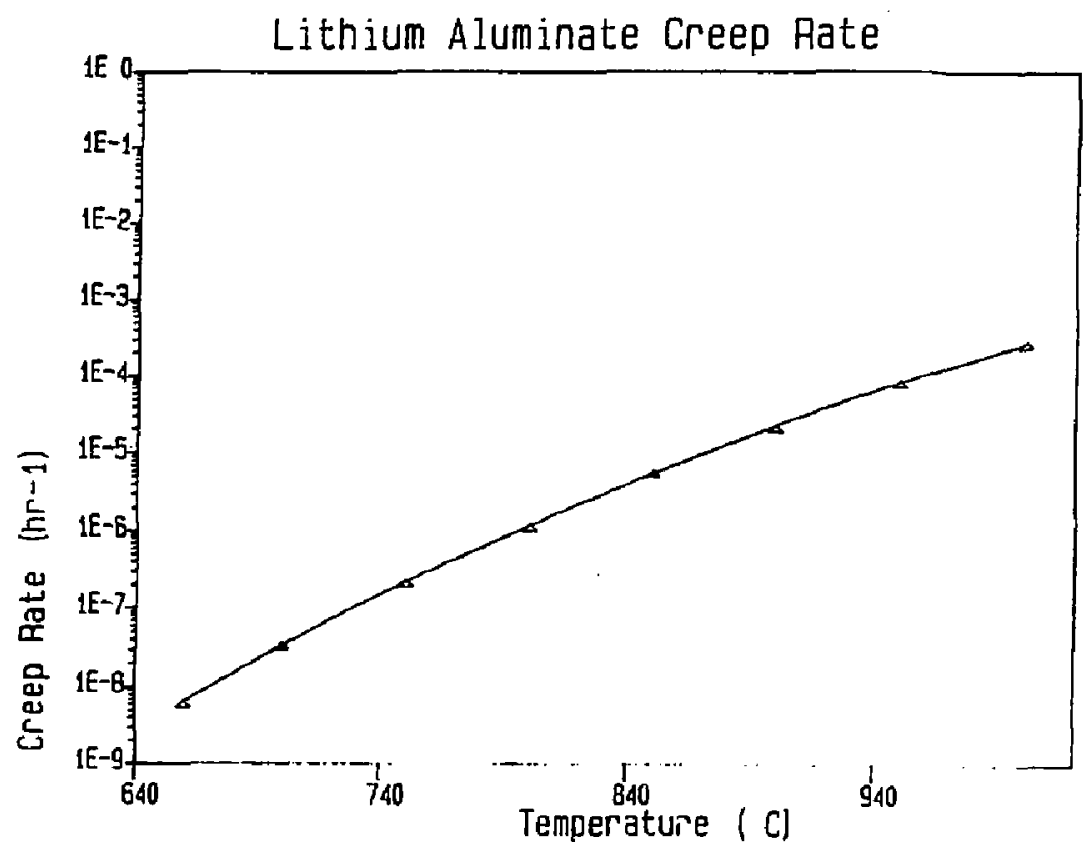




\section{PHENOMENOLOGICAL SWELLING EQUATIONS FOR SOLID}

BREEDER AND NEUTRON MULTIPLIER MATERIALS

\subsection{Theory}

The behavior of Solid Breeder Materials under irradiation has been the subject of various experiments. Hollenberg (2) conducted experiments on: $\mathrm{Li}_{2} \mathrm{O}, \mathrm{LiAlO}_{2}$ and $\mathrm{LI}_{4} \mathrm{SiO}_{4}$ ceranics th the FUBR-1A experiment in EBR-II. He repor:ad results on helium retention and swelling of these materials after $=18$ Li burnup $\left(\approx 3 \times 10^{20}\right.$ captures $\left./ \mathrm{cm}^{3}\right)$ as a function of irradiation temperature. Figure 1 shows the percent helium retentions in $\mathrm{Li}_{2} \mathrm{O}$ and $\mathrm{LiAlO}_{2}$.

In $\mathrm{Li}_{2} \mathrm{O}$. helium retention is a strong function of cemperature, in $\mathrm{IiAlO}_{2}$, however, almost no temperature dependence was detected. Furthermore compared to $\mathrm{Li}_{2} \mathrm{O}, \gamma-\mathrm{LiAlO}_{2}$ has very low helium retention capabilities. The cause of swelling is believed to be helium bubbles forming during irradiation. Thus the different recention rates of helium in $\mathrm{LiO}_{2}$ and $\gamma$-Lip. $\mathrm{O}_{2}$ manifest themselves into different swelling rates. Fig. 2 lends some qualitative support for the relationship between helium retentions and swelling. However, the low and athermal helium retention behavior in $\gamma-\mathrm{LiAlO}_{2}$ are not known at present. Microstructure examination is needed to ascertain the helium bubble morphology in order to explain experimental findings. Since it is believed that swelling has its microstructural origin in helium behavior, we will develop a simple swelling equation based on the following gas behavior assumptions:

* All retained gas is trapped in bubbles

* A11 bubbles are of the same size

* Van der Waal's Equation of State (EOS) is used

* Bubbles are in mechanical equilibrium with the solid 


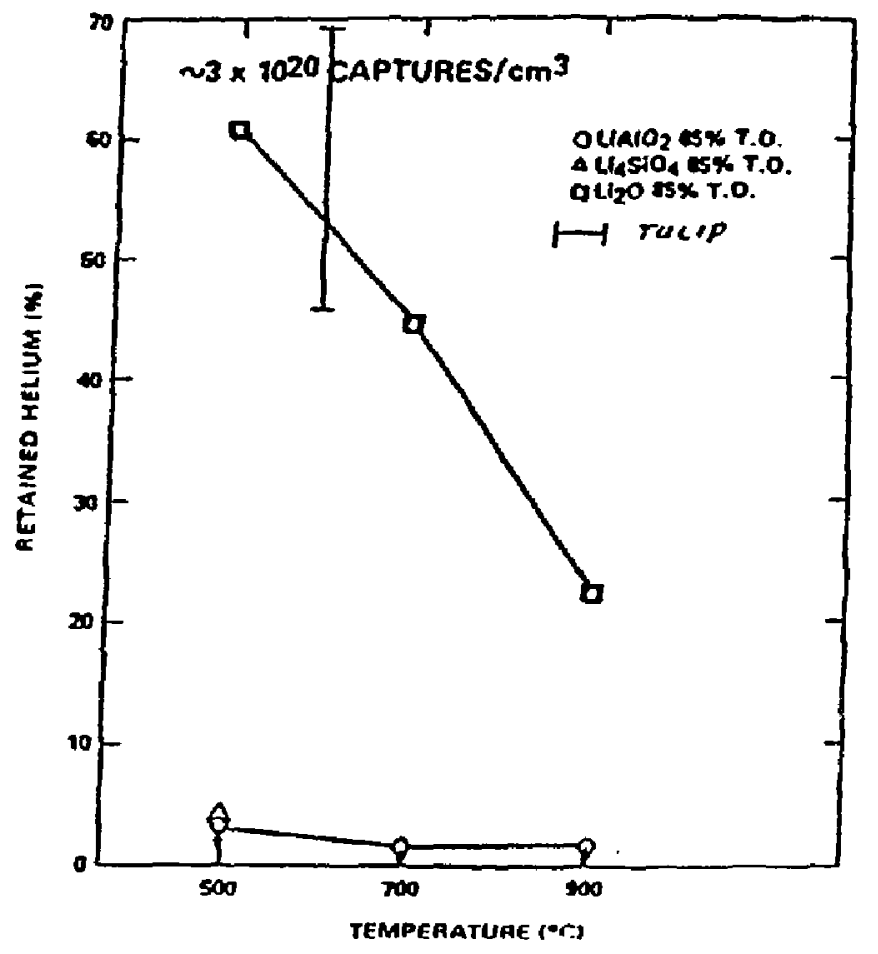

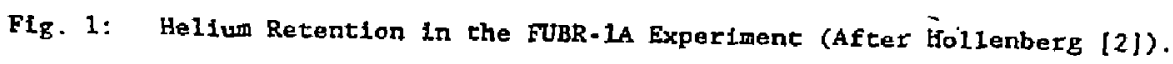




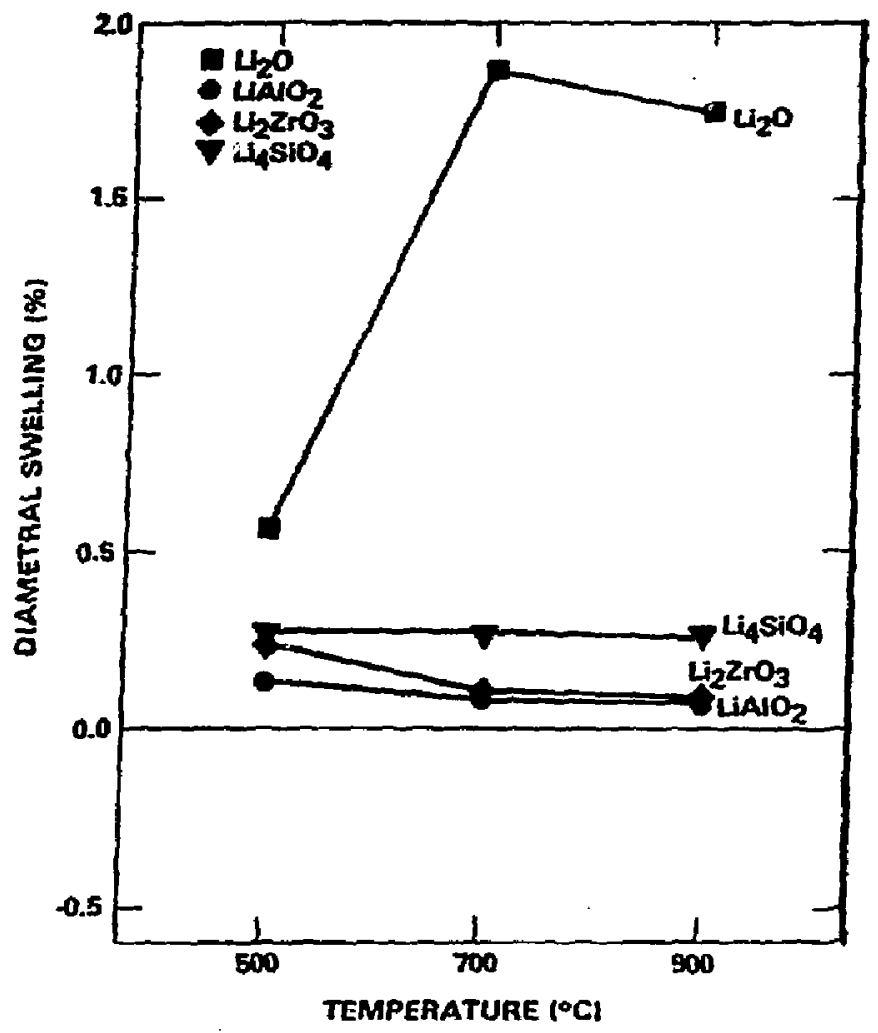

Fig. 2: Diametrical Swelling of $\mathrm{Li}_{2} \mathrm{O}, \mathrm{L}_{4} \mathrm{SlO}_{4}, \mathrm{LL}_{2} \mathrm{ZrO}_{3}$ and $\mathrm{LLALO}_{2}$ After Achleving a Burnup of $3 \times 10^{20}$ captures $/ \mathrm{cm}^{3}$ (After Hollenberg $\{2\}$ ). 
* Re-solution is neglected

The Van der Waal's EOS is most commonly used to describe the thermodynamic state of fission gases in bubbles. It can be written as

$$
P\left(\frac{I}{P_{g}}-B\right)=k T
$$

where $p$ is the pressure of a gas of molecular density $\mathrm{gg}$ at temperature $T$. The van der Waal's parameter $B$ can be regarded as an expression for the volume occupied by the gas atom. Assuming no hydrostatic stress, the mechanical force balance on a bubble dictates that:

$$
\mathrm{p}=\frac{2 y}{\mathrm{R}}
$$

where $\gamma$ is the surface tension of a bubble with radius $R$. EIiminating pressure between equation (1) and (2), we get for the gas density of a bubble:

$$
\frac{1}{\rho}_{g}-B+\left(\frac{k T}{2 \gamma}\right) R
$$

The number of gas atoms ( $m$ ) contained in a bubble of radius $R$ is

$$
m=\left(\frac{4}{3} \operatorname{IR}^{3}\right) \rho_{\mathrm{g}}
$$

or using Eq. 3

$$
m=\frac{4 \Pi^{3} / 3}{B+\left(\frac{k T}{2 \gamma}\right) R}
$$

If the functional swelling is small, we can express swelling $(\Delta V / V)$ due to gas bubbles by

$$
\frac{\Delta V}{V}-\left(\frac{4 \Pi R^{3}}{3}\right) N
$$

where $\mathrm{N}$ is the bubble number density. We are assuming that all retained hellum atoms are trapped inside bubbles of equal size. Thus we can relate 
the numbe of gas atoms per bubbles to $\mathrm{N}$ by

$$
\mathrm{f}_{\mathrm{R}} \hat{G}_{\mathrm{He}} \tau \cdot \mathrm{mN}
$$

where $f_{R}$ is the fraction of helium atoms retained and $\stackrel{\circ}{H e}_{H}$ is the helium production rate and $t$ is the time into irradiation. Combining equation (5), (6) and (7) we can express the swelling due to retained helium atams produced during irradiation time $t$ :

$$
\frac{\Delta V}{V}=\left[\frac{3}{4 \pi}\right]^{1 / 2}\left[\frac{\mathrm{kT} T}{2 \gamma} \frac{f_{\mathrm{R}} \dot{\mathrm{G}}_{\mathrm{He}} \mathrm{t}}{\mathrm{N}^{1 / 3}}\right]^{3 / 2}
$$

\subsection{Swelling of Solid. Breeder Material}

Thus we have an expression for swelling as a function of temperature, helium production rate and irradiation time. Note that swelling scales as $t^{1.5}$ which is commonly found experimentally.

To use eq. (8), knowledge about the helim number density is required. The experiment [2] provides us with three swelling data points. Using an approximate value of $\approx 1000 \mathrm{ergs} / \mathrm{cm}^{2}$ for bubble surface tension, we find from eq. (8) and the experimental value for swelling and helium retentian at $700^{\circ} \mathrm{C}$ a bubble number density of $\mathrm{N} \approx 1.5 \times 10^{-14} \mathrm{~cm}^{-3}$. Using Eq. 6, we can now estimate the average bubble radius at specified temperatures. Table 2 shows the estimaces for $N$ and $R$ using the experimental data points [2] . 
TABLE 2

Estimates for bubble number densities and radif using above model and experimental swelling data [2] for $\mathrm{LiAlO}_{2}$

\begin{tabular}{c|c|c|c}
$T(0)$ & $* \Delta V / V(8)$ & $R(A)$ & $N \times 10^{-14}\left(\mathrm{~cm}^{-3}\right)$ \\
\hline 500 & 0.12 & 192 & 0.382 \\
700 & 0.09 & 112 & 1.533 \\
900 & 0.07 & 69 & 5.017
\end{tabular}

* Experimental data

Assuming a temperature insensitive bubble number density for $500 \leq T \leq$ $900{ }^{\circ} \mathrm{C}$ we find a slight increase in swelling as a function of temperature, while experimental evidence points to a slight decrease. In Table 3 we compare experimental to analytical results.

TABLE 3

Experimental [2] and Aralytical [Eq. 8]

Swelling Results for $\mathrm{LiAlO}_{2}$ for $500 \leq \mathrm{T} \leq 900{ }^{\circ} \mathrm{C}$

\begin{tabular}{c|c|c|} 
& \multicolumn{2}{|c|}{$\Delta \mathrm{V} / \mathrm{V}(\$)$} \\
$\mathbf{T}\left({ }^{\circ} \mathrm{C}\right)$ & Exp. & Ana1 \\
\hline 500 & 0.12 & 0.015 \\
700 & 0.09 & 0.092 \\
900 & 0.07 & 0.122
\end{tabular}

We can now estimate swelling rates of $\mathrm{LiAlO}_{2}$ for our blanket conditions. As a demonstration of our model we can use a helium generation rate of about $5 \times 10^{13} \mathrm{He} / \mathrm{cm}^{3} / \mathrm{sec}$, typical for $5 \mathrm{~mW} / \mathrm{m}^{2}$ neutron wall loadings. Furthermore we assume a bubble number density of about $1.5 \times 10^{14} \mathrm{~cm}^{-3}$ and an athermal heliur recention fraction of 38 . Fig. 3 shows the results of our phenomenological equation. Under these conditions the swelling is estimated 


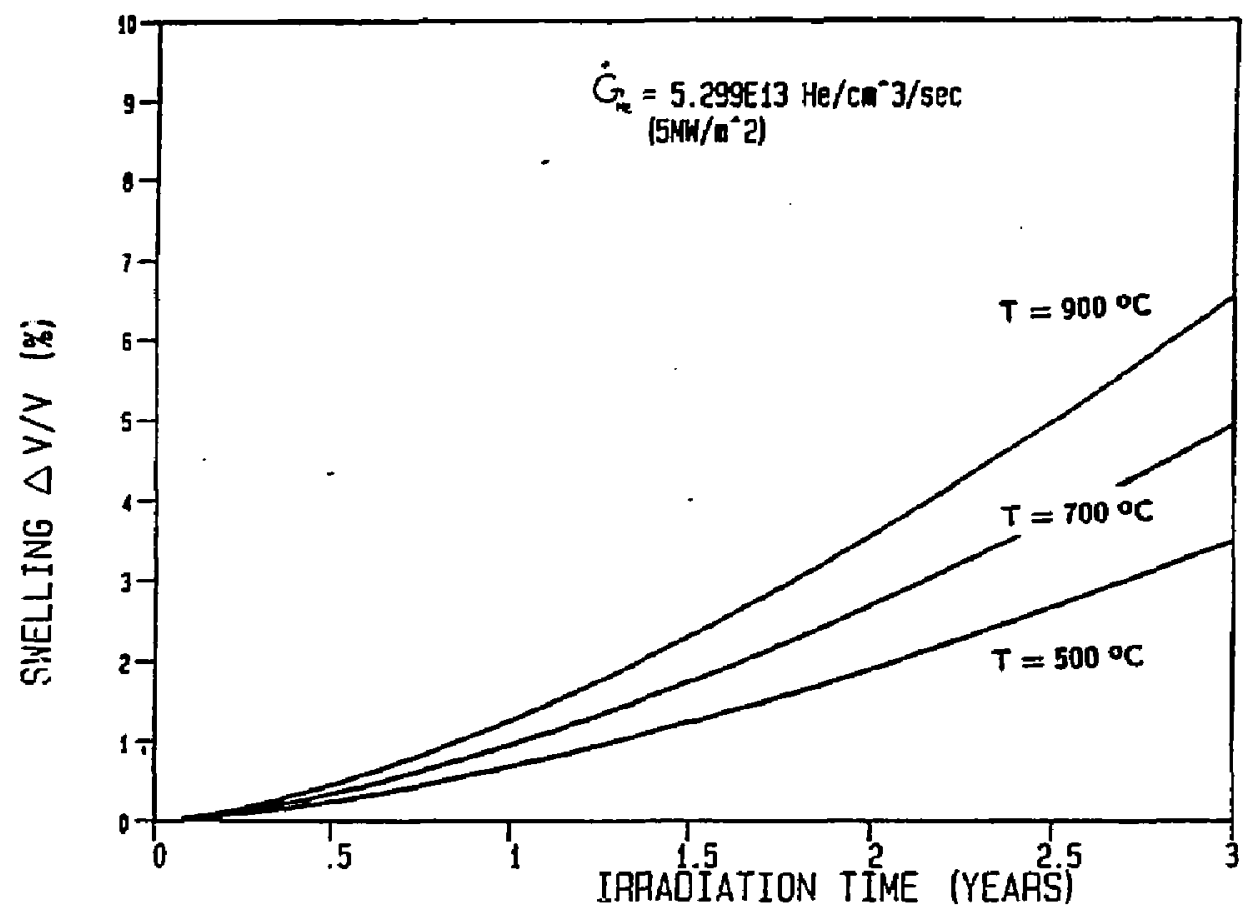

Fig. 3: Volwetric Swelling of $\mathrm{LiAlO}_{2}$ Exposed to $\approx 5 \mathrm{wW} / \mathrm{w}^{2}$ of Neutron Wall Loading is a Function of Irradiation Time. 
to $\approx 6.7$ at $900^{\circ} \mathrm{C}$ and $3.3 \%$ at $500^{\circ} \mathrm{C}$ after 3 years of irradiation.

Using the approach outlined above, we have also used Hollenberg's experimental swelling data for $\mathrm{Li}_{2} \mathrm{O}$ [2]. Table 4 shows the experimental findings for swelling and hellum retention at various temperacures.

\section{TABLE 4}

Experimental sweIling and helium retention data [2] for $\mathrm{Li}_{2} \mathrm{O}$

\begin{tabular}{c|c|c}
$\mathrm{T}(0 \mathrm{C})$ & $\Delta V / \mathrm{V}(\mathrm{8})$ & $\mathrm{f}_{\mathrm{r}}(\mathrm{g})$ \\
\hline 500 & 0.6 & 60 \\
700 & 1.8 & 43 \\
900 & 1.6 & 20
\end{tabular}

The modeI combined with these experimental data points allows us to estimate the bubble number density $(N)$ and bubble radii $(R)$. Table 5 shows these estimates.

TABLE 5

Estimate for bubble number densities and radif us in the above model and experimental data [2] for $\mathrm{Li}_{2} \mathrm{O}$

\begin{tabular}{c|c|c|c}
$I\left(\mathrm{C}^{\circ}\right)$ & $* \Delta V / \mathrm{V}(\mathrm{z})$ & $\mathrm{R}(\mathrm{A})$ & $\mathrm{N} \times 10^{15}\left(\mathrm{~cm}^{-3}\right)$ \\
500 & 0.6 & 61.5 & 6.19 \\
700 & 1.8 & 195 & 0.58 \\
900 & 1.6 & 323 & 0.12
\end{tabular}

* Experimental values

We see an increase in bubble radius as a function of temperature for $\mathrm{LI}_{2} \mathrm{O}$, while for $\mathrm{LAAlO}_{2}$ (Table 2) we note a decrease in bubble size. This is 
' consistent with the swelling results measured by Hollenberg [2]. In LiAlO 2 thermal emission of helium atoms from bubbles is probably the major ca:tse for bubble shrinkage thus leading to a decrease in swelling with incriasing temperatures. Our model does not reflect this behavicr because of the simplifying assumptlons we had to make due to lack of knowledge of helium behavior in cecamics. We are assuming constant size and constant bubble densities in describing the swelling behavior. These assumptions are more justified for $\mathrm{Li}_{2} \mathrm{O}$ ceramics since swelling more than doubles over the 500 to 900 'C temperature trange. Thus our model's predictions are in better agreement for $\mathrm{Li}_{2} \mathrm{O}$ than for $\mathrm{LiAlO}_{2}$. Table 6 compares our model result to experimental data.

\section{TABLE 6}

Experimental [2] and analytical [eq. 8] swelling resules for $\mathrm{Li}_{2} \mathrm{O}$ for $500<\mathrm{T}<900^{\circ} \mathrm{C}$

\begin{tabular}{c|c|c|}
\multirow{2}{*}{$\mathrm{T}\left({ }^{\circ} \mathrm{C}\right)$} & \multicolumn{2}{|c|}{$\Delta V /(8)$} \\
\cline { 2 - 3 } 500 & ExD. & Ana1. \\
\hline 700 & 0.6 & 0.58 \\
900 & 1.8 & 1.55 \\
& 1.6 & 1.55
\end{tabular}

Experimental data for $\mathrm{Li}_{2} \mathrm{O}$ show a highly temperature sensitive swelling rate and helium retention fraction. Because of this we no Ionger can assume constant bubble number densicles and ratention fractions without introducing large errors in the model. Because of this, we have used the values of Table 4 to evaluate swelling rates for $\mathrm{Li}_{2} \mathrm{O}$ at the various temperatures. The results of applying our swelling equation (eq. 8) to our reactor conditions are demonserated in FIg. 4. We find our model to follow experimencal trends predicting a silghtly higher swelling race for $700^{\circ} \mathrm{C}$ chan for 500 or $900{ }^{\circ} \mathrm{C}$. 


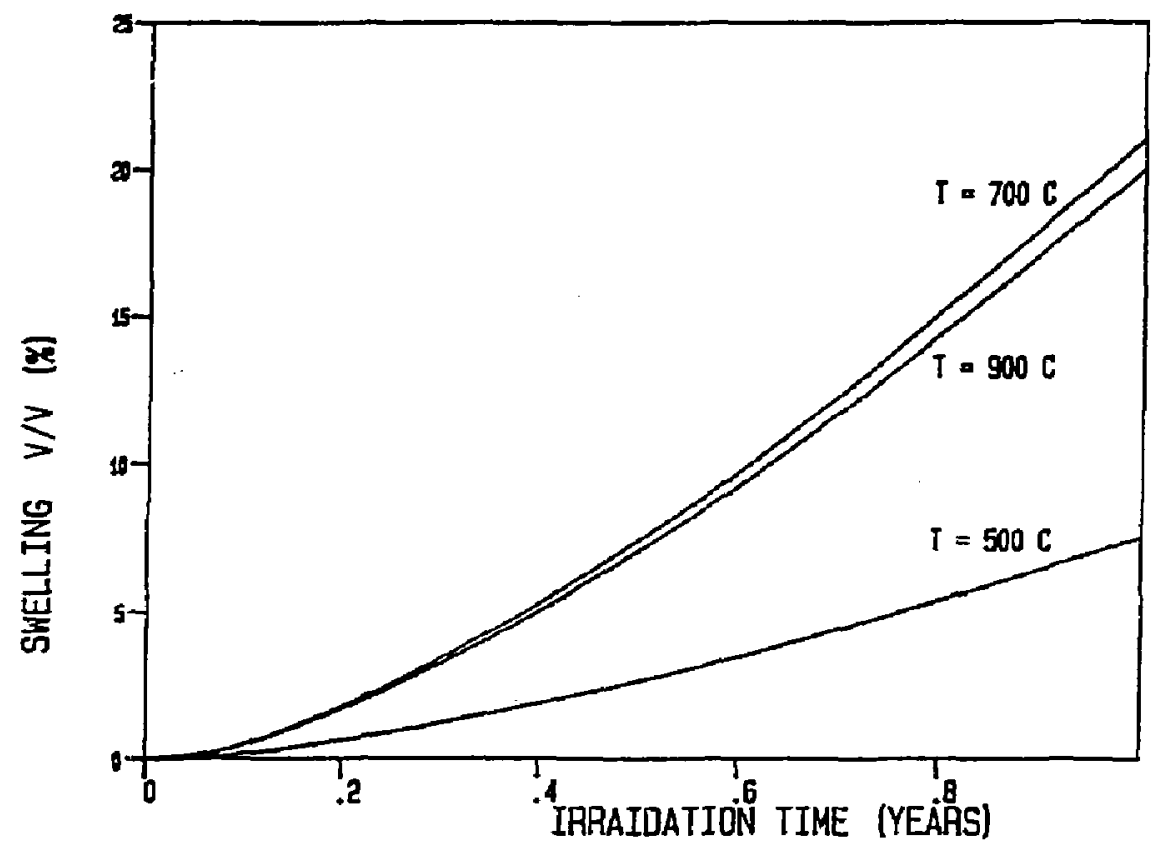

Fig. 4: Volumetric Swelling of Lij ${ }^{\circ}$ Exposed to a $5 \mathrm{MW} / \mathrm{m}^{2}$ of Neutron Wall Loading as a Function of Irradiation Time. 
For our model to be used at intermedfate temperatures between 500 and $700^{\circ} \mathrm{C}$, one could use a simple linear extrapolation of the bubble number densities and fraction of helium retained from Tables 4 and 5 .

It should be noted, however, that our approach is purely a phenomenological one, which does not necessarily take into account the actual physical behavior of helium atoms in ceramics under irradiation. Our model does not include important parameters such as porosity, grain size. dislocation network or irradiation produced damage. A model describing these phenomena would require a major effort combining experiment with basic analytical research over a period of many years.

\subsection{Beryllium Swelling}

The swelling equation (eq. 8) can also be applifed to betyllium. Beryliium has a large $(n, 2 n)$ reaction associated with helium production. Furthetmore, the $14 \mathrm{MeV}$ neutrons create high PKA energies. Because of these characteristics, swelling is of major concern in berytlium. Effects of helium production and irradiation-induced dimensional changes in Be have been the subject of experimental investigations using fission spectrums by Beeston and coworkers $[10,11]$. They irradiated Be-specimens in ETR and ATR up to $3.5 \times 10^{22} \mathrm{n} / \mathrm{cm}^{2}(\mathrm{E}>1 \mathrm{MeV})$. Beeston's et. al. $[10,11]$ measurements represent low neutron energy, low fluence irradiation conditions. Because the effects of fast neutron, high fluence irradiation are not known at the present time, we have to base our model on Beeston's data. Thus our simple model, although applicable to tission conditions, has to be used cautiously when applied to fusion conditions. As was the case before, we need to know the bubble number densities, the fraction of helium atoms retained and the surface energy of beryllium.

Beeston gives an empirical equation for the number density of helium 
bubbles for an annealing temperature between $400^{\circ}$ and $600^{\circ} \mathrm{C}$.

$$
\mathrm{N}=1.4 \times 10^{14} \exp (0.45 / \mathrm{kT}) \mathrm{cm}^{-3} \quad[9]
$$

No measurements for the fraction of helium atoms retained were reported. Thus we will cake the conservative approach of trapping all produced helium atoms inside the bubbles. Surface energy measurements quote values ranging from 1 to $2 \mathrm{~J} / \mathrm{m}^{2}[12,13,14]$. Wolfer et. al. [15] have also investigated beryllium swelling behavior. They [13] use a similar simple swelling model and report best agreement between model and data for a surface energy value of $2 \mathrm{~J} / \mathrm{m}^{2}$. Thus we will use the $2 \mathrm{~J} / \mathrm{m}^{2}$ value for the surface energy of beryllium in our model. Tabie 7 compares Beeston's data $(10,11]$ to our mode1.

\section{TABLE 7}

Experimenta1 $[10,11]$ and analytica1 swelling results for Be irradiated to 50,000 appm at $400^{\circ}<\mathrm{T}<600^{\circ} \mathrm{C}$

\begin{tabular}{c|c|c|} 
& \multicolumn{2}{|c|}{$\Delta \nabla /(8)$} \\
$T(\sigma \mathrm{C})$ & Exper. & Analyt. \\
\hline 400 & 5 & 4.54 \\
450 & 9 & 6.61 \\
500 & 12 & 9.24 \\
550 & 17 & 12.5 \\
600 & 20 & 16.4
\end{tabular}

We have applied the simple swelling model to the present blanket design. Fig. 5 shows the average pin temperature as a function of distance from the flist wall. The maximum Be-pin temperarure is $\approx 440^{\circ} \mathrm{C}$ while the $\mathrm{LAAlO}_{2}$ hotcest $\mathrm{pin}$ reaches $=1000^{\circ} \mathrm{C}$. The $\mathrm{six} \mathrm{Be}-\mathrm{pins}$ are all of the same 


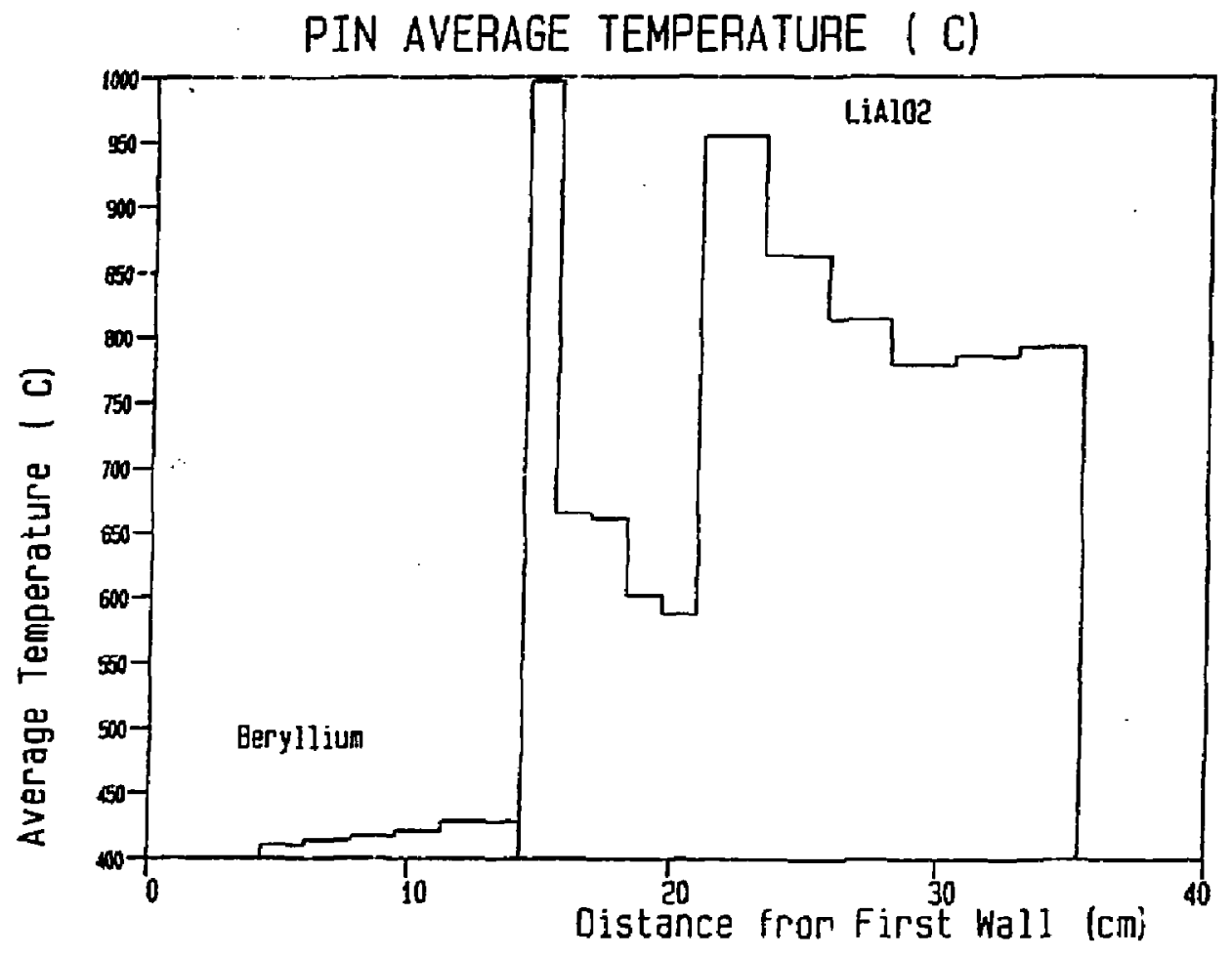

Fig. 5: Blanket Pin Average Steady Stace Temperature as a Function of Distance from the First Wall for a Neutron Wall Loading of a 5 $\mathrm{MW} / \mathrm{m}^{2}$. 
size. The five front $\mathrm{L}_{1 \mathrm{AlO}} \mathrm{O}_{2}$ pins are roughly half the size of the following. six rows of breeder pins. Fig. 6 shows the helium atom production rate in the $\mathrm{LiAlO}_{2}$ pins as a function of distance from the first wall. The helium atom production rate in the beryllium rods is depicted in Fig. 7. Note that the helium production rate is roughly an order of magnitude larger in Be than it is in the breeder pins.

In Fig. 8 we have shown the volumetric swelling of the $\mathrm{LiAlO}_{2}$ breeder pin at the end of three years. We have assumed a constant helium atorm production rate. Thus not allowing for burnup of lithium atoms. Nevertheless, the maximum swelling occurs in the front row of breeder pins with a top value of $=1.3$ after 3 years. This level of swelling would be tolerable for blanket lifetimes of 3 - 4 years.

The high belitum-generation rate in Be-rods turns out to be the life limiting phenomena for our blanket design. In Fig. 9 we have depleted the beryllium volumetric swelling at various irradiation times. After 1 year of irradiation the maximum swelling occurring in the first row of Be-rods reaches 7.78. This value increases to unacceptable limits of 20 and 308 after 2 and 3 years respectively. Although these levels of swelling seem to limit the blanket lifetime to about one year, one should note that no allowance was made for Be-burnup during the irradiation time. Furthermo:e, at high levels of swelling, microstructural evolutions will play an important role. High swelling rates coupled with thermal cycling lead to the formation and propagation of cracks. If bubbles form predominately on grain boundaries, Interlinkage of bubbles can lead to gas releases high enough to reach swelling peaks. Another important microstruccural evolution is the role of dislocation network formation during irradiation. Dislocations can sweep bubbles from the matrix to grafn boundaries, or act as gas cunnelling 


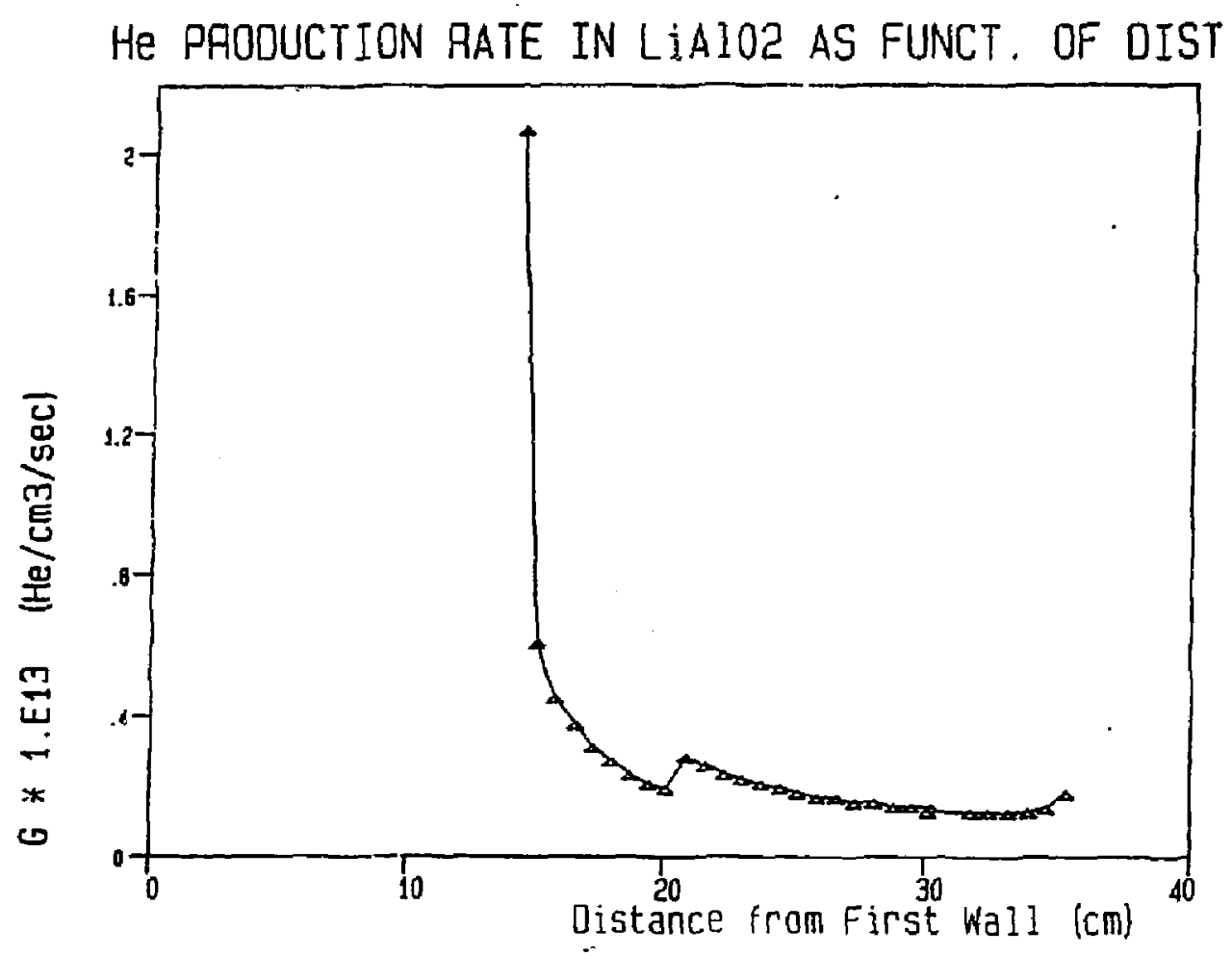

Fig. 6: Helium Production in $\mathrm{LIAlO}_{2}$ Breeder Pins as a Function of Distance from the First Wall. 


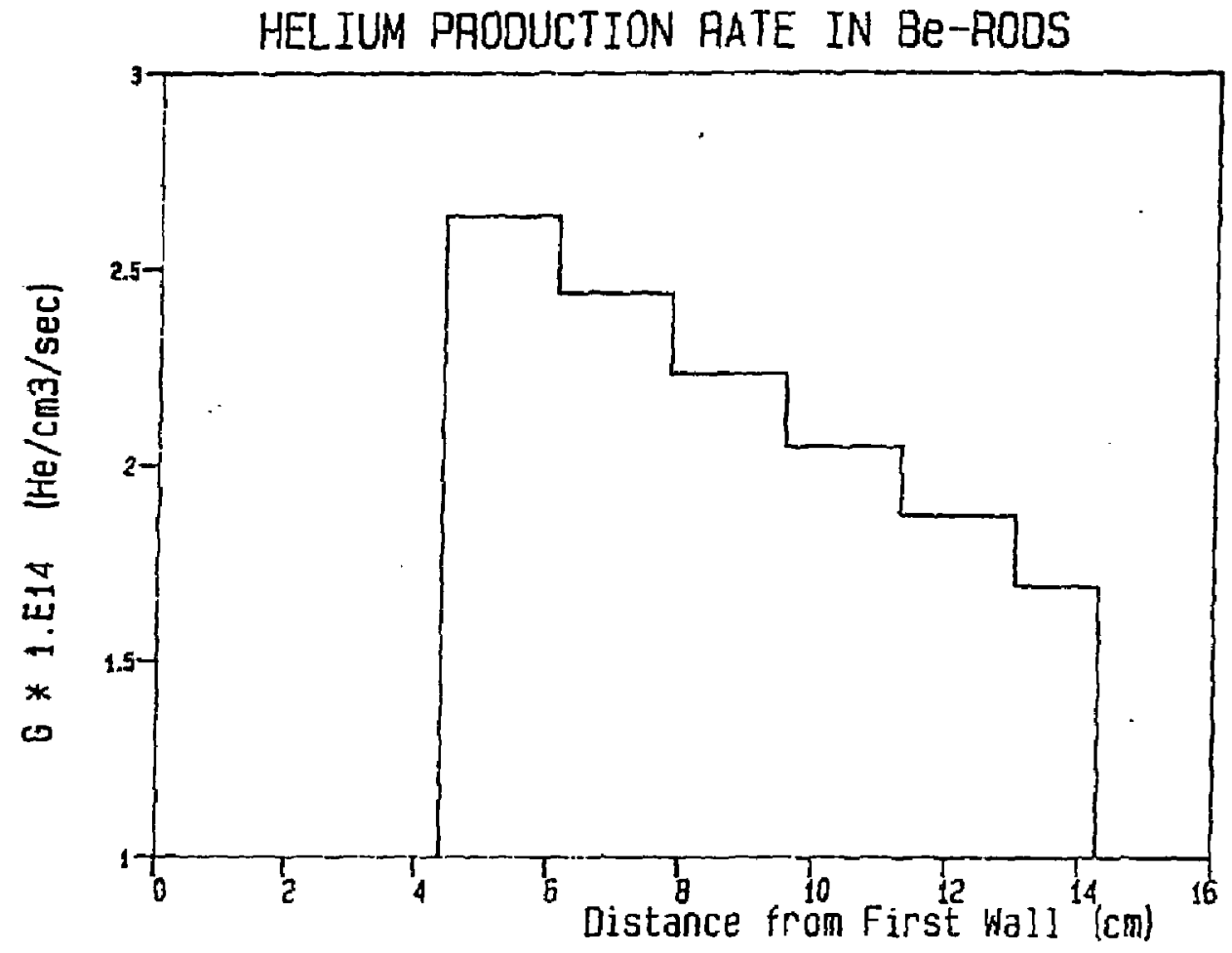

Fig- 7: Average Helium Production in Be-Rods as a Function of Distance from the First Vall. 


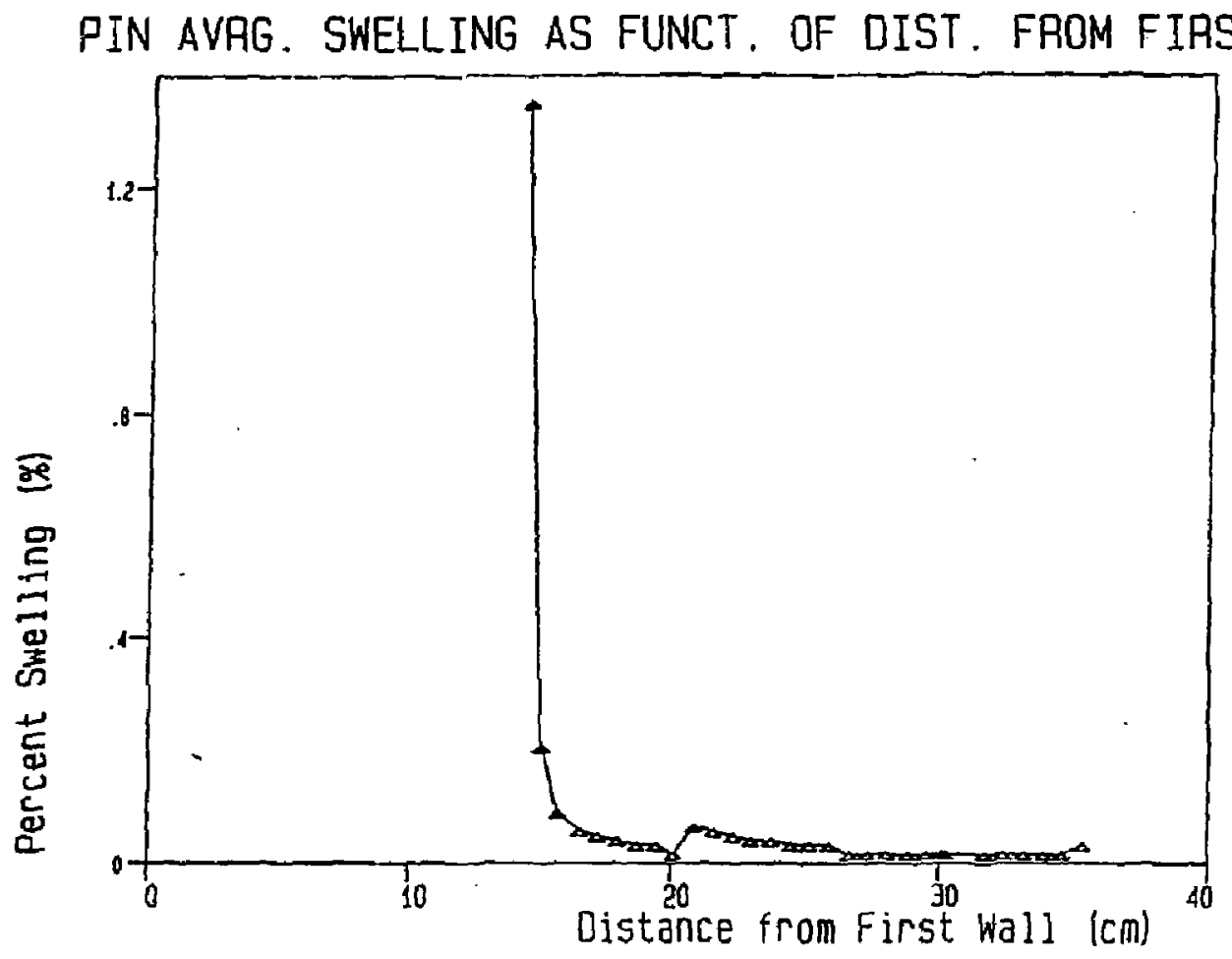

Fig. 8: Average Volumetric Swelling of $\mathrm{LiAlO}_{2}$ - Breeder Rods Exposed to 5 MW/ $/ \mathrm{m}^{2}$ Neutron WalI Loading after 3 Years of Irradiation. 


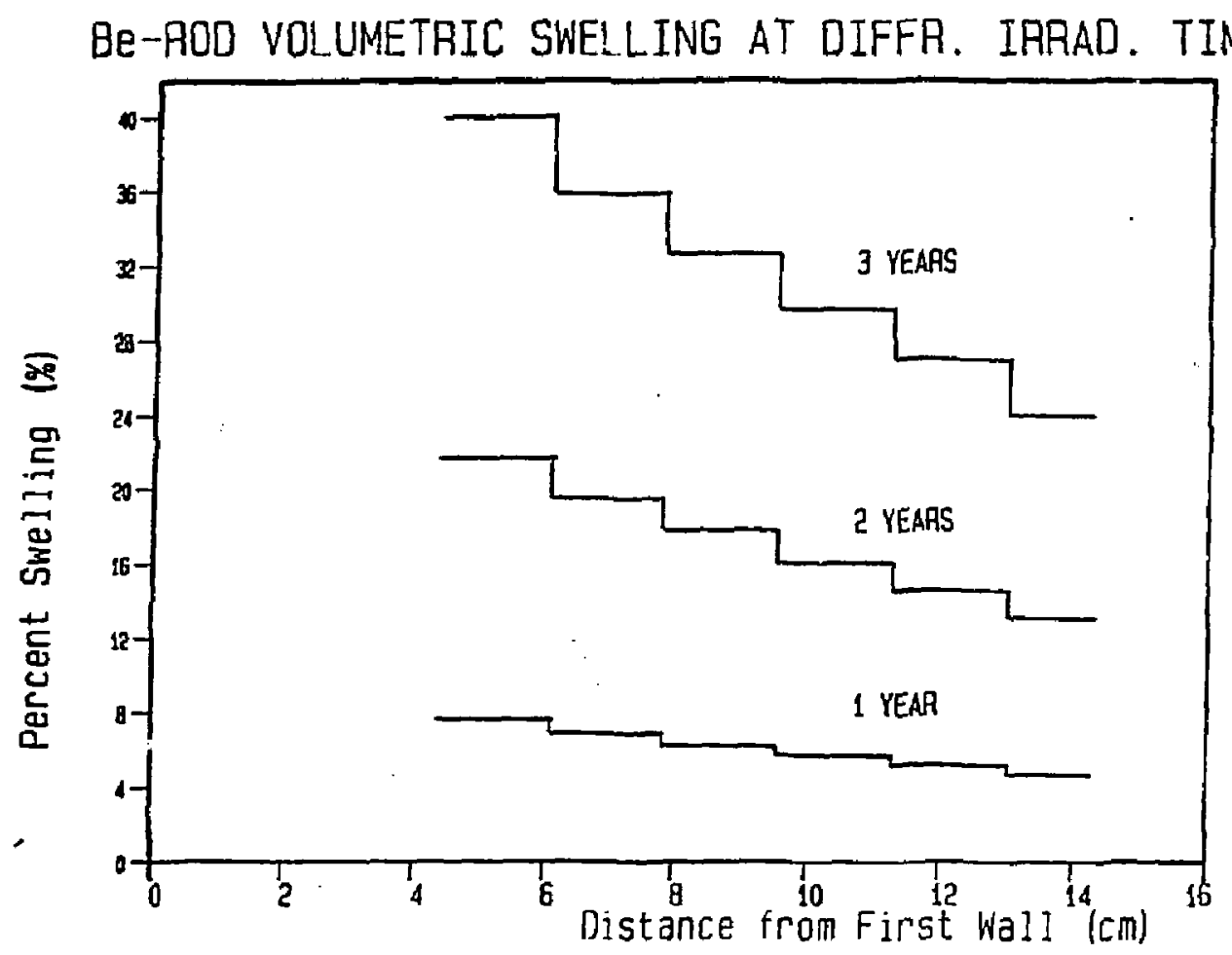

Fig. 9: Volumetric Swelling of Be-Rods Exposed to $5 \mathrm{MW} / \mathrm{m}^{2}$ Neutron Wall Loading after 1,2 and 3 Years of Irradiation. 
channe1s. Both phenomena lead to an increase of gas release from the matrix. Furthermore, dislocation climb is considered a major creep mechanism that can accommodate voluretric swelling. Due to lack of consideration of microstuctural phenomena, the high swelling rates for Berrods cannot be considered accurate. They only point to a potential problem which has to be investigated in great detail experimentally and analytically. 


\section{Sintering Phenomena in Breeder Ceramics}

\subsection{Intraduction}

Two configurations for solid breeders have been considered: pressed and sintered pellets and sphere-pac. Gold pressing and sintering techniques were developed for $\mathrm{Li}_{2} \mathrm{O}, \mathrm{Li}_{4} \mathrm{SiO}_{4}$, and $\gamma$-LiAlO 2 samples irradiated in ORR [16] and EBR-II irradiation experiments [17].

For IWR and fast reector fuel rods sphere-pac techpology has been developed and is considered attractive for solid-braeders.

The sphere-pac solid breeder configuration could potentially allow for higher thermal conductance due to the elimination of pellet clad gaps. This configuration isumes that internal shifting of the sphere-pac will accommodate expansions or contractions of solid breeder material.

A sound understanding of the factors that govern the temperature distriburion within the solid-breeder material is essential to successful prediction of breeder performance over long periods of time and in an Entense radiation field.

The temperature distribution, which depends strongly on porosity. influences solid-breeder performance in two important ways:

1. High cemperatures: Solid-state reactions that would be immeasurably slow at lower temperatures proceed at rates sufficient to produce significant changes in material properties during the life-time of the breeder in the reactor.

Phenomena primarily affected by high operating temperatures include:
a. Grain growth
b. Densification (sintering) 
c. Fission-product diffusion

2. Staep tenperature gradients: High temperatures can close pores thich nigrate up a temperature gradient; important constituents of solid-breeder macerial, such as oxygen, $\mathrm{LI}$, and ffasion producta, are redistributed from their initial concentration profiles (whlch are ugually uniform); chermal stresses resulting from the temperature gradients cause breeder material aither to deform plastlally in regions of high temperature or to crack in low-temperature zones.

Bojause porosity determines both temperature and temperature gradients in solfd breeders, the next section will outline some effects of porosity.

The following section looks at the posstbility of sinterfing of sphere-pac solid breeder material.

\subsection{The Effect of Porosity}

Oxide fuel. 3 are generally fabricated by sintering pellets of pressed powdered $\mathrm{VO}_{2}$ or mLxed $\mathrm{UO}_{2}-\mathrm{PuO}_{2}$ at high temperatures, (typically $1700{ }^{\circ} \mathrm{C}$ ) for a predetermined length of time. By control of the sintering conditions, materials of any desired density between 80 and $90 z$ of theoretical denstty can be produced.

In the sphere-pac route, high density ceramic spheres of controlled sizes are produced and then subsequently loaded with the assistance of low-energy vibration.

The high denstity ceramic spheres are produced in three steps:

1. Preparation of a spectal solution

2. Gelation of droplats to gfve semf-rigid spheres

3. Drying and sintering these spheres to a high density. 
Inasmuch as porosity in a ceramic body invariably reduces its thermal conductivity, it would appear desirable to eliminate all internal pores or voids tn the fuel fabrication process.

However, $a$ certain amount of fabricated porosity is useful in accommodating the fission products that accumulate during irradiation; porosity is a means of minimizing fuel swelling. The density of both thermal and fast reactor fuels are well below theorecical value. The porosity of the fast reactor $\left[(\mathrm{U}, \mathrm{Pu}) \mathrm{O}_{1.96}, \rho=908\right.$ TD] is purposely made greater than that of the thermal reactor fuel $\left[\mathrm{UO}_{2+x}=928 \mathrm{TD}\right.$;, because of the larger burmups required in the former. (Larger buriugs are required to enhance breeding-ratios

Since controlled soltd breeder material porosity is a design variable, it is important to be able to predict the effect of porosity on solid-breeder properties, In particular on thermal conductivity and consequenty fission gas $\left({ }^{3} T\right)$ diffusivity.

Theoretical analysis of the effect of porosity on thermal conductivity has been hampered by the number of variables that must be considered. One variable that appears in most theorecical models is the volume porosicy (P), defined by:

$$
\text { E - } \frac{\text { valume of pores }}{\text { volume of pores }+ \text { volume of solid }}
$$

In addition, the geometry and physical properties of the individual pores may also be importanc. Pore geometry is defined by its size, shape and orientation with respect to the direction of heat flow. Physical properties that may be significant ate the emmisivity of the solid and the thermal conductivity of the gas trapped within the pore (if any).

In 19:4, Loeb [18] treated the thermal conductivity in a manner that permitted many of the secondary varlables mentioned in the preceding 
paragraph to be properly accounted for. In 1966, Biancheria [19] was able to theoretically account for the effects of pore shape.

The well-known Loeb equation describing conductivity of a porous material is:

$$
k=k_{s}(1-P)
$$

where $k$ - thermal conductivicy of a pozous material

$k_{s}$ - thermal conductivicy of a solid material

P - porosity given by

$$
P=1-\left(\frac{g}{s}\right)
$$

where $\stackrel{\rho}{\rho}_{s}$ is percentage of theoretical density.

Ineb's equation has been found to underestimate the porosity effect on the thermal conductivity of $\mathrm{UO}_{2}$. This deficiency has been remedied by Inserting an adjustable parameter to yield:

$$
k=k_{s}(1-\alpha P)
$$

Equation (11) is called the modified Loeb equation. Values of the parameter a from 2 to 3 have been determined by fitting this equation to $\mathrm{UO}_{2}$ thermal-conductivity measurements.

Biancheria's [19] analysis of the porasicy effect yield the following formula:

$$
\frac{\mathrm{k}}{\mathrm{k}_{\mathrm{s}}}=\frac{1-\mathrm{P}}{\mathrm{I}+(\alpha-1) \mathrm{P}}
$$

However, contzary to the purely empirical nature of the parameter $\alpha$ in equation (II), \& in equation (12) can be evaluated for equal-sized pores of a particular geometry randomly distributed in the solid. For spheres 
a - 1.5; for axisymmetric shapes such as ellipsoids of revolution, the sphere factor $\alpha$ is greater than 1.5.

In theory, the thermal conductivity of porous ceramics can be determined by using equations 9,11 or 12 . However, extensive measurements of $\mathrm{vO}_{2}$ thermal conductivity have shown that the theoretical formulas do not agree well enough with experfments to serve as design equations .

This trend will probably hold true for solid-breeder material and purely empirical formulas should be used when reliable numerical values are needed.

Yung Y. LIu, M.C. Billone and R. Clemmer [20] have performed thermal-conductivity measurements on ifradiated and unirradiated Li ${ }_{2} \mathrm{O}$-sintered pellet and sphere-pac $\gamma$-LiAlO ${ }_{2}$, which could be utilized to compare to theoretical values and to produce empirical equacions for $k_{e f f}$ of $\mathrm{Li}_{2} \mathrm{O}$ and $\mathrm{y}-\mathrm{LiAlO}_{2}$.

\subsection{Sintering}

Lithium oxide pellets in the form of cylinders with 1 in. in diameter by $1 \mathrm{in.}$. in height have been prepared for the Tilk lithium blanket module (LBM) program [21] .

To facilitate the release of bred tritium, the density of these pellets is purposely kept well below theoretical densities (TD). Takahash1 and Kikuchi [22] have demonstrated that as the density of $\mathrm{Li}_{2} \mathrm{O}$ pellets is increased above 868 , the Eraction of closed porosity remains essentially constant at 38 to $4 \%$ of the pellet volume.

Sphere-pac requires three sizes of spheres to achieve abour $88 \%$ smear density. If a lower density is acceptable, two sizes will yield 
about 858 smear density [23].

The contact area between adjacent microsphere plays an important role in the thermal conductivity. Although there is only a $=3$ s difference in density between ternary and binary mixtures of $\mathrm{vo}_{2}$, experiments [24] show that the effective conductivity of the ternary mixture of $\mathrm{UO}_{2}(2.75 \mathrm{w} / \mathrm{m}-\mathrm{k})$ is much higher than that of a binary mixture $(0.9 \mathrm{w} / \mathrm{m}-\mathrm{k})$.

Fuel-gap conductance has also been measured for sphere-pac fuel and pellets of Identical fuel composition [25]). These measurements show a gap conductance of $7.3 \mathrm{~kW} / \mathrm{m}^{2}$ o $\mathrm{C}$ for pellet fuel and $19.3 \mathrm{~kW} / \mathrm{m}^{2}{ }^{\circ} \mathrm{C}$ for sphere-pac fuel. This reduction of contact resistance between the breeder and its clad can significantly change temperature distributions of the breeder material.

From the point of view of thermal conductivity and lithium loading in the blanket, the density of the solid breeder material should be maximized, whereas from the point of view of tritium recoverability porosity, in particular open porosity, should be maximized.

One of the challenges encountered in solid-bteeder material design lies in determining this optimum density for the breeder marerial. Once determined, what will the upper temperature limit be to insure no further densification?

To shed light on some of these issues let us examine some sintering mechanisms which could occur in sphere-pac solid breeder material.

During sintering, an originally porous compact is transformed inco a dense ceramic. Most commonly pores become more spherical (i.e., closed) In shape and smailer in size as firing continues. The free energy that gives rise to densification is the decrease in surface area and lowering 
of the surface free energy by the elintngtion of solld-vapor interfaces. Thls usially takes place with the colncidental fornation of new, but loter-energy, solfd-solfd incerfaces. Thus rescructuring of the contact area betweon the microsphere causes a change in shape and shrinkage of the pore.

The different mechanisms for material tranffer durlng sintering processes are:

a. Evaporation and Condensation

b. Viscous flow

c. Surface dtffuston

d. Grain-boundary or Lattice diffusion

e. Plastic deformation.

In treating fission fuel waterial the wo mechanfsas shown to be 1mportant are: Evaporation-Gondensation and Diffusion. In the absence of temperature gradients vapor phase material transfer can occur due to vapor pressure differences as a function of particle curvatures.

For micron-range particle size the vapar gressure has to be larger than $10^{-4}$ - $10^{-5}$ atm for evaporation-condensation to be significant.

Vapor pressures reach signiflcant values for $\mathrm{LI}_{2} \mathrm{O}$ above $1200^{\circ} \mathrm{G}$ and for $\mathrm{LIAlO}_{2}$ above $1400^{\circ} \mathrm{C}$, both wall above proposed upper temperature limits.

However, if vapor pressures are low, material transfer may occur more rapidiy by diffusion. Diffusion is equivalent to the migration of lattice vacancies in the opposite direction. Material can migrate from the grain boundaries between particle to the neck area between two microspheres resulting in equivalent wotion of particle conters teward one mother. This results in simultaneous changes in pore size and pore 
shape and a corresponding decrease in overall porosity.

Kingerly [26] has derived an expression for the rate of growth of bond formation $(x)$. His finding, $x \propto e^{1 / 5}$, are verified experimentally.

$$
\frac{x}{r}=\left[\frac{40 \gamma D \Omega^{3} n^{1 / 5}}{k T}\right]^{-3 / 5} t^{1 / 5}
$$

Using equation (13), Kingerly [26] derived an expression for the shrinkage of a compact:

$$
\frac{\Delta V}{V}-3\left[\frac{20 \gamma n^{3} D_{v}}{\sqrt{z k T}}\right]^{2 / 5} r^{-6 / 5} t^{2 / 5}
$$

From this expression he concluded that shrinkage is not strongly time dependent $\left[(\Delta V / V) \propto \tau^{2.5}\right]$ but size dependent. The sintering rate is roughly proportional to $\mathrm{r}^{-1}\left[(\Delta V / V) \propto \mathrm{r}^{-6 / 5}\right]$; as the particle size is decreased, the rate of sintering is raised.

The other variable affecting sintering races is che vacancy diffusion coefficient which is sensitive to temperature and impurities. Because the vacancy diffusion coefficients have not been well extablished for solid-breeder material use of Kingerly's equations can be misleading in determining sintering behavior of $\gamma$-LAAlO

Some experimental measurements [27] indicate the onset of sintering In sphere-pac $\gamma-\mathrm{LLAlO}_{2}$ around $1200^{\circ} \mathrm{C}$.

Great uncertainty remains as to the effect of irradiation on the sintering rate.

The kinetics of the sinterlng process depends on the location of the pores (uhich are the vecancy sources) relative to the grain boundaries (which are the vacancy sinks). Porosity close to the grain boundaries is 
more rapidly eliminated than porosity in the middle of the grain for in the latter case vacancles must diffuse through the bulk.

The rate of shrinkage of a porous solid is governed by the flux of vacancles reaching the grain boundary from the pores.

in Kingery's model, the only means by which vacancies left a pore and entered the adjacent solid was by diffusion.

In solid-breeder material exposed to radiation, however, vacancies can be jjected into the solid by the disruptive action of primary-knock on-atoms (PKA's) passing through or near a pore. This is a type of re-solution process generally associated with fission-gas bubbles. Although a pore can be thought of as consisting of $(4 / 3) \mathrm{HR}_{\mathrm{p}}^{3} / \Omega$ vacancies, the vacancies are not ponderable particies that can acquire kinteic energy by collision with high-speed particles (e.g., a fission fragment or a latcice knock-on). Therefore, pore shrinkage by fission fragnents or PKA's must be described by a form of macroscopic re-solution, in which a passing fragment blasts solid from one side of a pare to the other, trapping some acancies in the deposited side in the process. The buried vacancies are considered to be redissolved and to acquire the mobility of single vacancies in the lattice.

Because the pores in ceramic fuels and thus in solid-breeder material are considerably larger than fission-gas bubb?es, it is unlikely that a pote can be completely converted to vacancies by a single re-solution event. Hence re-solution is considered to reduce the size of the pores but not to change the total pore concentration. In sphere-pac solid breeder material we have highly dense microspheres with the porosity concentrated in large volumes between the spheres. In this case it is even more unlikely that re-solution will have any effect on the 
shape or size of the pores and consequently on the sitering rate.

The other irradiation effect that could be considered is the substantial vacancy production rate due to colliston cascades and fission spikes. In densification models neither of the vacancy sinks in the solid (grain boundarles) exhibits a preference for interstitials or vacancies; so an excess of one type of point defect in the solid cannot be built up. Only dislocatlons are biased sinks, and they are not considered. If they were, swelling rather than shrinkage would probably occur, for this is the prime effect of the presence of dislocation sinks $\because r$ void swelling in metals.

The above discussion shows that unrealistic assumption concerning the distribution of the pores, their inttial size, the neglect of grain growth concurrent with densification, and the effects of radiation on re-solution and pore shape have to be made in order to model sintering of solid-breeder materlal.

Therefore, we have to rely on experiments to set guide-lines in the development of solid-breeder material.

\section{Acknowledgement}

This work was supported by the U.S. Department of Energy, Office of Fusion Energy, Grant \#DE-FGG3-80ER52061, to UCLA. 


\section{References}

[1] Materials Handbook for Fusion Energy Systems, DOE/TIC-10122; Apr 11 30, 1980; John W. Davis, Coordinator

[2] G,W. Hollenberg, "Fast Neutron Irradiation Results on $\mathrm{Li}_{2} \mathrm{O}$, $\mathrm{Li}_{4} \mathrm{SiO}_{4}, \mathrm{Li}_{2} \mathrm{ZrO}_{3}$ and $\mathrm{LiAlO}_{2}{ }^{n}$, J. Nuclear Materials 122\&123 896-900 (1984).

[3] B. Rasneur, "Trittur Breeding Material: $\gamma$-LiAlo ${ }_{2}$, Fusion Technology, Val. 8, July 1985.

[4] R.J. Amodeo and N.M. Ghoniem, J. Nuclear Materials; Vol. 122 , Nos. 1-3; May 1984, p. 91.

[5] R.J. Amodeo and N.M. Ghoniem, "Development of Design Equations for Ferritic Alloys in Fusion Reactors", NED/Fusion 2 (1985) 97-110.

[6] D.S. Gelles and R.J. Puigh, "Alloy Development for Radiation Performance", Semi-Annual Progress Report, DOE/ER/0045/11 Sept. 1983).

[7] N.M. Ghoniem and R.W. Conn, Assessment of Ferritic 3teels for Steady State Fusion Reactors", International Atomic Energy Agency, Proc. on Fusion Reactor Design and Technology, IABA-TG-392/62, 701. II (1983) p. 389.

[8] R.J. Amodeo and N.M. Ghoniem, Development of Design Equations for Ferritic Alloys in Fusion Reactors: NED/Fusion 2 (1985) 97 - 110

[9] D.S. Gelles anil R.J. Puigh, Alloy Development for Radiation Performance, Semi-Annual Progress Report, DOE/aR/0045/11 (Septembr: 1983)

[10! J.M. Beeston, M.R. Martin, C.R. Brickman, G.E. Korth, and w.C. Francis, in Symp. on Materials Performance in Overating Nuclear Systems, Ames Laboratory, Ames, Iowa, August 1979, Nuclear Metallurgy 19, CONF-730801, Pp. 59-87.

[11] J.M. Beeston et al., "Comparison of Compression Properties and Swelling of Beryllium Ir:adiated at Various Temperatures," EGG-FT-6608, Idaho National Engineering Laboratory (1984).

[12] R.S. Barnes and G.B. Redding, Nuclear Energy 10, p.22, 1959,

[13] J.B. Rich, G.B. Redding and R.S. Barnes, J. Nucl Matls. I, p. 73, 1959.

[14] L.E. Muir, Interfacial Phenomena in Metals and Alloys, Addison-Wesley Publ. Corp., Reading, MA, 1975, p. 124.

W.G. Wolfer and T.J. McGarville, "Swelling of Beryllium", DAFS, 
DOE/ER-0046/19, Nov. 1984 .

[16] L. Yang, R. Hedico, W. Baugh, and $\mathrm{K}$. Schultz, "Irradiation Study of Lithium Compound Samples for Tritium Breeding Application," J. Nucl. Materlals, 103, 585 (1981).

[17] Program Review Documents of the Task Force on Material and Irradiation Effects for Solid Breeders During 1980-I983, coordinated by C.E. Johnson, ANL.

[18] A.L. Loeb, J. Amer, Ceram. Soc, 37:96 (1954).

[19] A. Biancheria, Trans. Amer. Nuc1, Soc., 9:15 (1966),

[20] Y.Y. Loeb, M.C. Billone, R. Glemer, "Solid Breeder Tritium Recovery," Blanket Comparison and Selection Study, March 20-21, 1984.

[21] "TFTR Lithium Blanket Module (LBM) Program, Freliminary Design Report," GA Technologies Report, GA-G16616, December 1981.

[22] T. Takahashi and T. Kikuchi, JAERI-M 7518 (1978).

[23] "Ge1-Spere-Pac Fuel for Thermal Reactor -- Assessment of Fabrication Technology and Irradjation Performance," ORNL-5469, November 1979 .

[24] M.J. Ades, "Thermal Conductivity of Sphere-Pac Fuel," Exxon Nuclear Company Report, DOE/ET/34215, Juiy 1981.

[25] A.L. Iotts, Comp., "Fast Breeder Reactor Oxide Fuels Development .. Final Report, ORNL-49-1 (November 1973).

[26] W.D. Kingerly, "Introduction to Geramics," John Wiley \& sons, Ine. 196?.

[27] G.W. Hollenberg, Private Conversation. 